\title{
Macroscopic urban dynamics: Analytical and numerical comparisons of existing models $^{\text {tis }}$
}

\author{
Guilhem Mariotte $^{\mathrm{a}, *}$, Ludovic Leclercq ${ }^{\mathrm{a}}$, Jorge A. Laval ${ }^{\mathrm{b}}$ \\ ${ }^{a}$ Univ. Lyon, ENTPE, IFSTTAR, LICIT, F-69518, Lyon, France \\ ${ }^{b}$ School of Civil and Environmental Engineering, Georgia Institute of Technology, Atlanta, GA 30332, USA
}

\begin{abstract}
Large-scale network modeling using the Macroscopic Fundamental Diagram (MFD) is widely based on the singlereservoir model, where the variation of the accumulation of circulating vehicles in the reservoir equals inflow minus outflow. However, inconsistent lags for information propagation between boundaries may be observed with this single accumulation-based model. For example, outflow is reacting too fast when inflow varies rapidly, whereas this information should be carried by vehicles that are never driving faster than the free-flow speed. To overcome this limitation, a trip-based model has been recently proposed, but whose solution cannot be obtained analytically.

In this paper we compare both models under piecewise linear MFD and a piecewise constant demand. These assumptions allow to establish the exact solution of the accumulation-based model, and continuous approximations of the trip-based model at any order using Taylor series. Moreover, a flexible event-based simulation framework is implemented to solve the latter model, making it a promising tool to account for heterogeneity in distance traveled. Thanks to these resolution schemes we are able to measure the inaccuracy of the accumulation-based approach when the demand varies rapidly, and propose a validity domain for this model. Other applications with different trip lengths and supply limitations are also discussed.
\end{abstract}

(c) 2017. The authors. This manuscript version is made available under the CC-BY-NC-ND 4.0 license

(cc) BY-Nc-ND http://creativecommons.org/licenses/by-nc-nd/4.0/

Keywords: Macroscopic modeling, single-reservoir, trip length, analytical resolution, piecewise linear functions

\section{Introduction}

Large-scale network modeling based on the Macroscopic Fundamental Diagram (MFD) has advanced significantly in recent years. It appears as a viable option for congestion management applications such as perimeter control (see e.g. Haddad and Geroliminis, 2012; Aboudolas and Geroliminis, 2013; Ramezani et al., 2015; Haddad, 2017) and for modeling large cities based on the multi-reservoir framework presented in Hajiahmadi et al. (2013); Knoop and Hoogendoorn (2014); Yildirimoglu et al. (2015); Kouvelas et al. (2017). The theoretical foundations of all these approaches have been established in Daganzo (2007); Geroliminis and Daganzo (2007), where the dynamics of a single reservoir is represented by a conservation equation where outflow is determined by the MFD function. This approach will be referred to as the "accumulation-based MFD model". Though it has proved to be an attractive description of large urban areas, this approach still relies on strong hypotheses, which may have non negligible impacts. For example, Xue et al. (2016) show that simplifying an urban system by two reservoirs may lead to a different optimal perimeter-control strategy than when the system is described using classical traffic flow theory. Also, it has been shown in Leclercq et al. (2015) that the accumulation-based model suffers from significant numerical viscosity even when the time step is small. Outflow (respectively inflow) may then overreact to sudden demand surge (respectively

\footnotetext{
DOI: 10.1016/j.trb.2017.04.002. Available online at www.sciencedirect.com

${ }^{*}$ Corresponding author. Tel. : +33 (0) 472047769

Email address: guilhem.mariotte@ifsttar.fr (Guilhem Mariotte)
} 
supply drop) leading to inconsistent propagation of information between opposite perimeter boundaries. The simplest illustration is an empty reservoir and a demand that starts increasing. The outflow instantaneously also increases creating an immediate reaction that may be interpreted as information that propagates from one boundary to the other at an infinite speed.

Another crucial point concerns the representation of vehicle trip length within the reservoir. It is often assumed constant for all vehicles for the sake of simplicity (see e.g. Haddad and Geroliminis, 2012; Aboudolas and Geroliminis, 2013; Hajiahmadi et al., 2013), but this is not consistent with what is observed when the local dynamics are taken into account (Leclercq et al., 2015). Trip lengths not only depend on the OD (Origin-Destination) matrix but also on the traffic conditions within the reservoir. Yildirimoglu and Geroliminis (2014) first highlight the error made in simulation when using the standard formulation of Daganzo (2007) in comparison with an improved description taking into account the variability of the trip length with respect to OD pairs. Such a description requires that the original single conservation equation that describes the behavior of the reservoir must be split into different vehicle classes, for example per OD pairs, with constant travel distances. The influence of the trip length for the accumulation-based MFD model is thoroughly investigated in Mariotte and Leclercq (2016). Leclercq et al. (2015) propose an alternative modeling framework to Yildirimoglu and Geroliminis (2014) to consider different trip lengths by defining macroscopic routes and jointly solving the related system of conservation equations. Based on an idea of Arnott (2013) and Fosgerau (2015), Daganzo and Lehe (2015) and then Lamotte and Geroliminis (2016) elaborate a simple and elegant reformulation of the single-reservoir dynamics to address the question of variable trip lengths. This reformulation will be further referred to as "trip-based MFD model" since the main idea is to guaranty that all vehicles cover their travel distance within the reservoir by adjusting their instantaneous speed to the current reservoir mean speed defined by the MFD.

In this paper, we compare the accumulation-based and the trip-based MFD models by investigating their analytical and numerical solutions for a piecewise linear MFD and piecewise constant inflows. The analytical solutions of the accumulation-based MFD model can be determined piece by piece while the trip-based MFD model require a continuous approximation based on the entering vehicle discretization. A major insight of our analytical developments is that the accumulation-based has no memory while the trip-based approach accounts for a reaction time. The comparison of the solutions on simple test cases will permit to further analyze the different model properties. In all cases, the trip-based approach appears superior as it better represents wave propagation and travel time evolution within the reservoir. On the other hand, an attempt to define a validity domain for the accumulation-based model application has been proposed. Numerical schemes are also derived for both approaches. Notably, we propose here an event-based solution method for the trip-based model that provides the exact solution when the inflow cumulative curve is kept as a step-function related to the entry of each individual vehicles. This solution method can be easily extended to account for different trip lengths for each driver, which is a significant improvement compared to the accumulation-based model. This means that the trip-based MFD model would be even easier to implement with multiple reservoirs.

The layout of this paper is as follows: we first present the analytical and numerical resolution of the accumulationbased reservoir model. Then we introduce the numerical resolution of the trip-based model and compare its properties with the original model. The event-based numerical scheme for the trip-based approach is also presented there. Finally, we compare the results with the previous method and the accumulation-based model on three case studies: a typical peak-hour demand profile, a supply restriction at the reservoir exit, and a peak demand case with various trip lengths inside the reservoir.

\section{Accumulation-based approach}

We present in this section the seminal accumulation-based approach for describing the dynamics of a singlereservoir system.

\subsection{The single-reservoir dynamics}

The single-reservoir system dynamics has been extensively presented in Daganzo (2007); Geroliminis and Daganzo (2007). The basic principle is that an urban network can be described in an aggregate manner with a reservoir characterized by its accumulation $n(t)$, the number of circulating vehicles at time $t$ (in [veh]). The traffic state within 
the reservoir is given by a well-defined relationship between the travel production $P$ (in [veh.m/s]) and the accumulation $n(t)$. Note that the mean speed $V$ of travelers (in [m/s]) is given by $V(n(t))=P(n(t)) / n(t)$ at every time $t$ (Geroliminis and Daganzo, 2007). As in Lamotte and Geroliminis (2016), $P(n)$ will be referred to as the "productionMFD", and $V(n)$ as the "speed-MFD". According to Geroliminis and Daganzo (2007), the trip length $L$ is the same for all travelers and satisfies the following equation:

$$
O(n)=\frac{P(n)}{L}
$$

where $O(n)$ is the reservoir outflow (in [veh/s]). It may represent either trips ending in the reservoir or trips exiting the area. This equation can be viewed as the application of Little's formula at the reservoir scale (Little, 1961). In this paper, we first add some further but non restrictive assumptions in order to facilitate analytical calculations. The initial state of the reservoir is free-flow so that the outflow is not restricted by the supply at the network exits. The total demand $\lambda(t)$ for internal or passing-by trips is always below the network capacity and therefore not limited by the reservoir entrance or supply function. With these hypotheses the evolution of the accumulation $n(t)$ is given by:

$$
\frac{d n}{d t}=\lambda(t)-O(n(t))
$$

Moreover we choose a piecewise linear shape for $P(n)$, in which each branch $P_{i}$ of $P(n)$ is defined by equation 3 :

$$
P_{i}(n)=w_{i}\left(n-\eta_{i}\right)
$$

where $w_{i}$ is the slope of the branch $P_{i}$ (in [m/s]) and $\eta_{i}$ the $n$-intercept (in [veh]), see figure 1(c). This choice is motivated by the analytical method in Leclercq et al. (2014). This assumption is not restrictive as any MFD can be approximated by such a family of linear envelope curves. Our analytical solution also considers that the demand $\lambda(t)$ is piecewise constant and thus the cumulative demand count curve is piecewise linear.

\subsection{Analytical solution}

Here we focus on the accumulation $n(t)$ and the experienced travel time $T(t)$, see figure 1(b). While accumulation evolves on the same branch $P_{i}(n)$ of the production-MFD and the demand remains constant, $\lambda(t)=\lambda_{0}$, the conservation equation 2 becomes:

$$
\frac{d n}{d t}=\lambda_{0}-\frac{w_{i}}{L} \cdot\left(n(t)-\eta_{i}\right)
$$

which corresponds to a first-order linear differential equation with constant coefficients. With the initial condition $n\left(t_{0}\right)=n_{0}$ at a given time $t_{0}$, the solution of $n(t)$ is:

$$
n(t)=\left(n_{0}-n_{\infty}\right) \mathrm{e}^{-\left(t-t_{0}\right) / \tau}+n_{\infty}
$$

where $\tau=L / w_{i}$ is the characteristic time of the system, and $n_{\infty}=\tau \lambda_{0}+\eta_{i}$ is the asymptotic value of $n(t)$ when $t \rightarrow+\infty$.

In this study $T(t)$ is the travel time experienced by the vehicle that exits the reservoir at time $t$. It corresponds to the time spent by the vehicle in the reservoir. The travel time evolution results from the model dynamics and can be determined from the integration of the outflow function, see figure 1(b).

The accumulation can be written as $n(t)=N_{\text {in }}(t)-N_{\text {out }}(t)$, where $N_{\text {in }}(t)$ and $N_{\text {out }}(t)$ are the cumulative count curves for entering and exiting vehicles respectively. Knowing that under FIFO $N_{\text {out }}(t)$ equals to $N_{\text {in }}(t-T(t))$ by definition, this gives directly $n(t)=\int_{t-T(t)}^{t} \lambda(s) d s=\lambda_{0} T(t)$; see figure 1(b). Therefore the travel time is directly obtained by dividing equation 5 by $\lambda_{0}$ :

$$
T(t)=\left(T_{0}-T_{\infty}\right) \mathrm{e}^{-\left(t-t_{0}\right) / \tau}+T_{\infty}
$$

where $T_{0}=n_{0} / \lambda_{0}$ is the initial travel time at $t=t_{0}$ and $T_{\infty}=n_{\infty} / \lambda_{0}$ is the asymptotic value of $T(t)$ when $t \rightarrow+\infty$. Note that this equation is only valid if the demand remains constant during $T(t)$ at least, otherwise the general relation $n(t)=\int_{t-T(t)}^{t} \lambda(s) d s$ must be used. The application of such a formula is straightforward as $\lambda(t)$ is piecewise constant. 

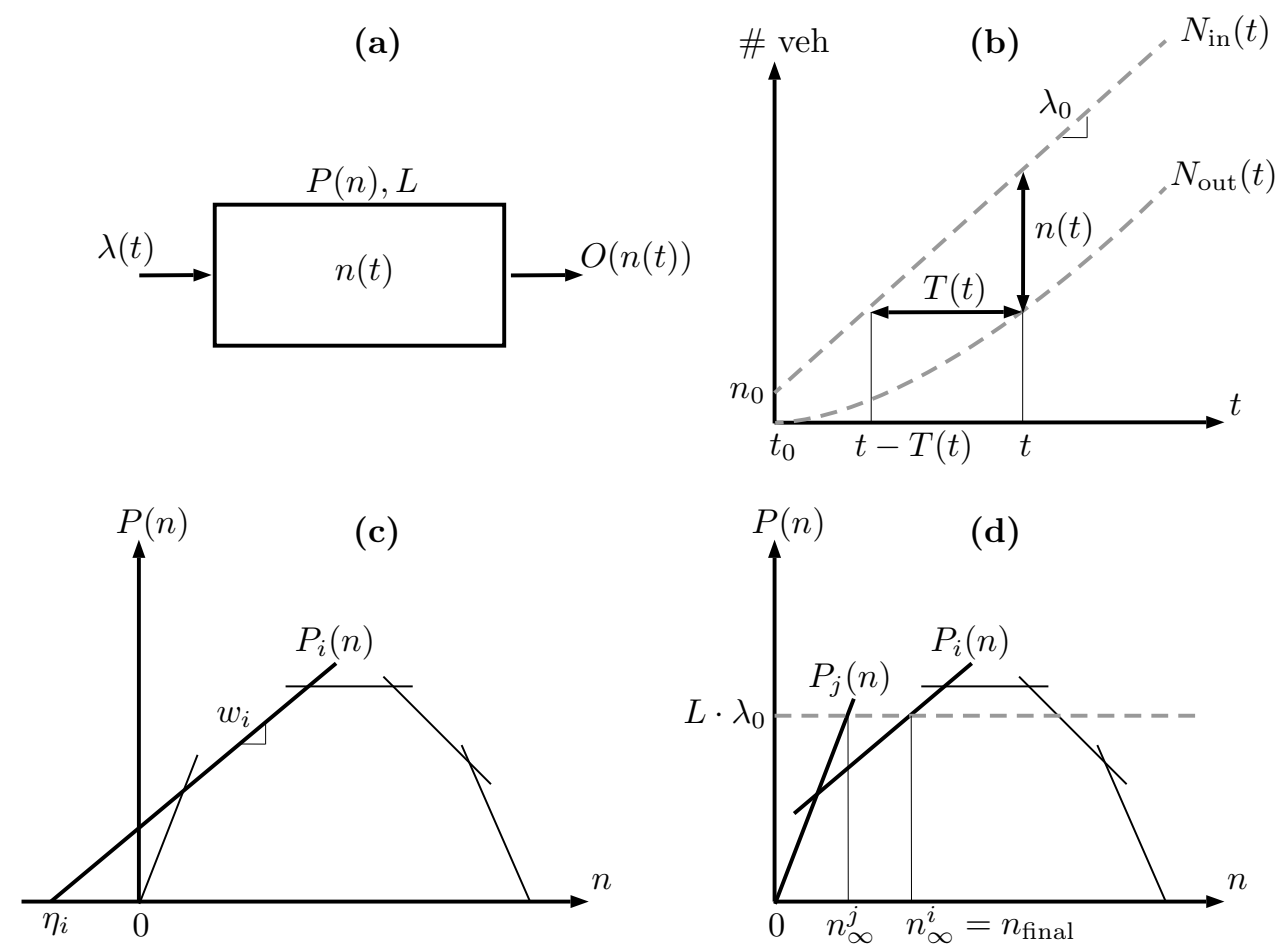

Figure 1: (a) Schematic representation of the single-reservoir dynamics, (b) entering and exiting curves with the definition of $n(t)$ and $T(t)$, (c) piecewise linear production-MFD $P(n)$, and (d) example of two successive applications of our analytical formula with a given demand $\lambda_{0}$ (transition from the MFD branch $P_{j}$ to $P_{i}$ )

\subsection{Step-by-step analytical resolution in the general case}

Because our initial condition in accumulation might take any value, for a given problem implying more than one branch of the piecewise production-MFD and different levels of demand evolving with time, a complete solution can be obtained by successive applications of the previous formulas in equations 5 and 6 . Note that our method can adapt to any demand variations since these are represented by step functions, and that this scheme does not require steady state to be reached before the next change in demand. Its principle is the following:

1. Initialize $t_{0}=0, n_{0}=n_{\text {ini }}$ (the initial accumulation for the whole problem)

2. Save the current level of demand $\lambda_{0}$ and directly calculate the entering vehicle curve $N_{\text {in }}(t)$ until the next change in demand

3. Determine the current branch of the production-MFD $\left(w_{i}, \eta_{i}\right)$

4. Update $n(t)$ and $T(t)$ using equations 5 and 6 until the minimum $t_{\min }$ between the time $t_{d}$ of the next change in demand and the time $t_{i}$ at which there is a change in the production-MFD branch. The latter can be determined by: $t_{i}=\tau \cdot \ln \left(\frac{n_{0}-n_{\infty}}{n_{i}-n_{\infty}}\right)+t_{0}$, where $n_{i}$ is the accumulation at the branch change defined in the production-MFD. Note that just after a change in demand the expression of $T(t)$ must be calculated using the general relationship $n(t)=\int_{t-T(t)}^{t} \lambda(s) d s$ as mentioned before.

5. Update the initial condition: $t_{0}=t_{\min }, n_{0}=n\left(t_{\min }\right)$. If $t_{\min }=t_{i}$, a change in MFD branch happens, go to 3 . Else if $t_{\min }=t_{d}$, a change in demand happens, go to 2 .

It is worth mentioning that during the warm-up period, i.e. when $N_{\text {out }}(t)<n_{\text {ini }}$ at the beginning of the simulation, we need additional hypotheses to define properly the travel time-in particular we need to know the previous demand level $\lambda_{\text {ini }}$ at $t=0$, see point 4 of the process. To this end we suppose that the system is in a steady state before $t=0$ characterized by the initial accumulation $n_{\text {ini }}$. For $t<0$ we have then $n_{\text {ini }}=\lambda_{\text {ini }} T_{\text {ini }}$ where $T_{\text {ini }}$ is the initial travel time equal to $L / V\left(n_{\text {ini }}\right)$. This yields the demand level that should be defined for $t<0: \lambda_{\text {ini }}=n_{\text {ini }} V\left(n_{\text {ini }}\right) / L$. 
With our step-by-step process any part of the global solution can be obtained at any precision. This resolution scheme provides by construction the exact solution of the accumulation-based model under our hypotheses.

\subsection{Application to a simple demand step case}

Figures 2(a) and (b) show a simple case of a one-staircase demand profile and a six-branch piecewise MFD, describing a 5-km network. The trip length is set to $L=2.5 \mathrm{~km}$, the free-flow speed to $u=15 \mathrm{~m} / \mathrm{s}$, and the maximum production to $P_{c}=3000 \mathrm{veh} . \mathrm{m} / \mathrm{s}$. The initial number of vehicles $n_{\text {ini }}$ has been chosen to correspond to the initial value of demand, so that the system is in steady state at $t=0$. These numerical values as well as the chosen MFD shape will be the same for all the numerical applications in this paper. Although the instantaneous increase in demand at time $t_{d 1}=700 \mathrm{~s}$ is rather unrealistic, this simple profile reveals a first drawback of the accumulation-based model: the analytical solution for the travel time exhibits a drop of $T(t)$ right when the demand increases. This phenomenon has also been noticed by Lamotte and Geroliminis (2016). The accumulation-based formulation in equation 2 does not include any features that bound or more precisely delay outflow variations when demand changes are observed. Hence an increase of the inflow leads to an instantaneous modification (increase) of the outflow, see figure 2(e). It means that information (changes of the flow between the reservoir entry and exit perimeter) is traveling between boundaries at a much higher speed than the maximal vehicle speed, and this information speed is even infinite at the time when the inflow changes. Travel time has to adapt to this immediate variation of the outflow to guaranty vehicle conservation. That means some vehicles have to drive faster to sustain the outflow rate, which explains the decrease of the travel time function at time $t_{d 1}$.

Figure 3 presents the evolution of accumulation and travel time for different initial conditions $n_{\text {ini }}$ and different levels of demand $\lambda_{0}$. Here the system is in steady state defined by $n_{\text {ini }}$ for $t<0$, and directly enters in transient state at $t=0$ because $\lambda_{0} \neq \lambda_{\text {ini }}$. The same non-intuitive drops in travel time are encountered. They are all the result of too fast propagation of the information between the reservoir boundaries. Figure 2(f) illustrates this problem by exhibiting wave propagation within the reservoir. Waves are here defined similarly as kinematic waves in link traffic flow theory, i.e. as locus in the $(x, t)$ plane where identical flow (or speed) values may be observed. Such kinematic waves represent the propagation of information in traffic stream as a change in flow value at one particular location will then propagate following such curves. Classically information is propagating forward in free-flow (positive kinematic wave speed) and backward in congestion. For reservoir models, there is no explicit representation of the $x$-axis but we can define wave by analogy to the link traffic flow theory by connecting identical flow values observed as inflow and outflow, i.e. between the two boundaries of the reservoir that define the starting and ending locations of trips. This permits to investigate how information is propagating inside the reservoir. Figure 2(f) clearly shows that some waves are faster than the vehicle speed (and even tends towards infinity when time tends towards $t=t_{d 1}$ ), because the outflow starts being modified before the first vehicle experiencing the demand surge joins the exit. This does not mean that some vehicles experience zero travel time because we are speaking here about the propagation of information. More precisely, only an infinitesimal small quantity of vehicles will indeed carry such a super-fast (and even infinite) information propagation. However, these inconsistent waves and output modifications induce the drop in travel times. This is why it is currently admitted in link traffic flow theory that waves should not travel faster than vehicle speeds. We believe that such a requirement is also important for reservoir models and we will show that the trip-based model in section 3 overcomes this problem. 
(a)

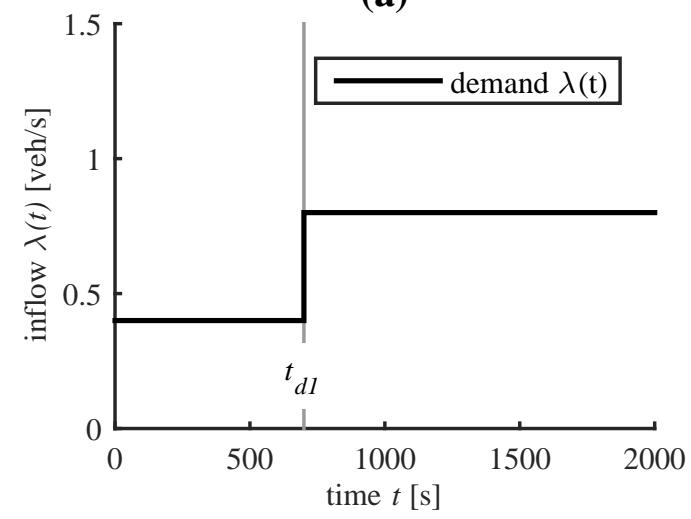

(c)

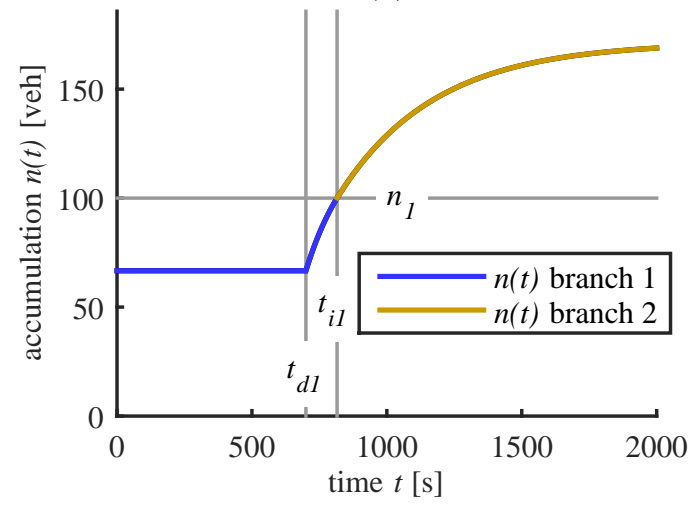

(e)

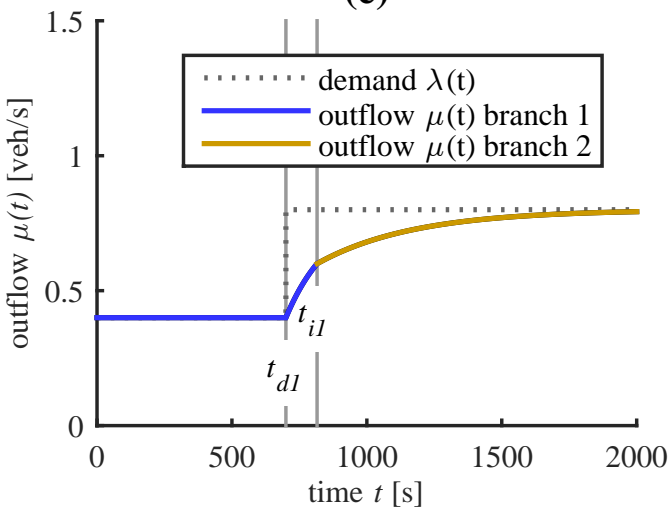

(b)

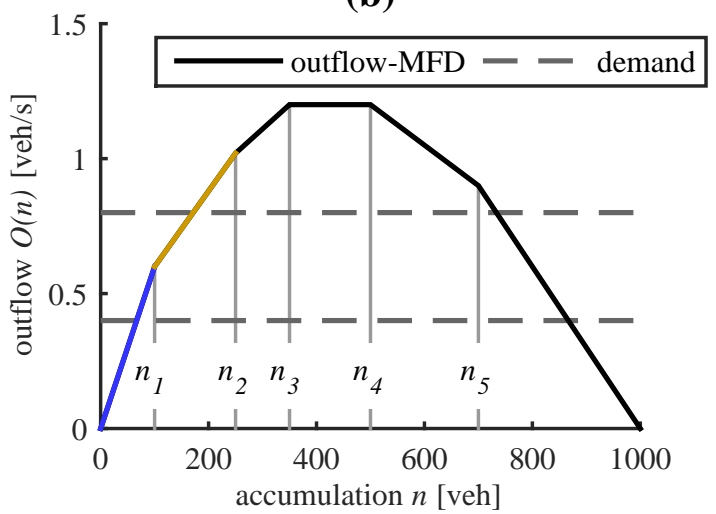

(d)

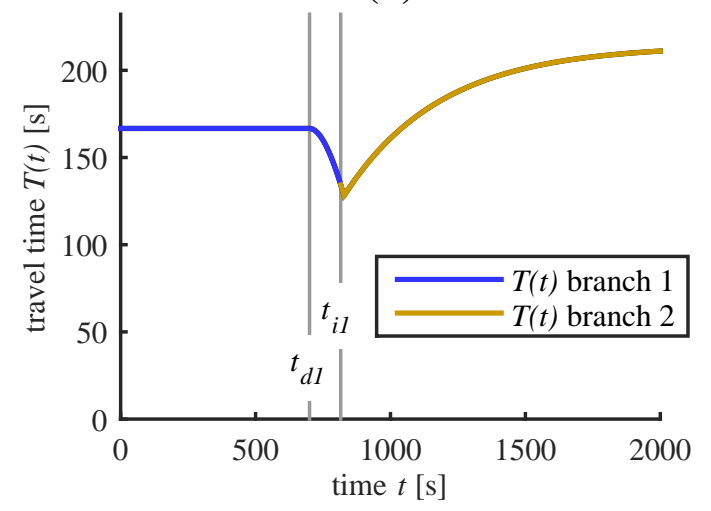

(f)

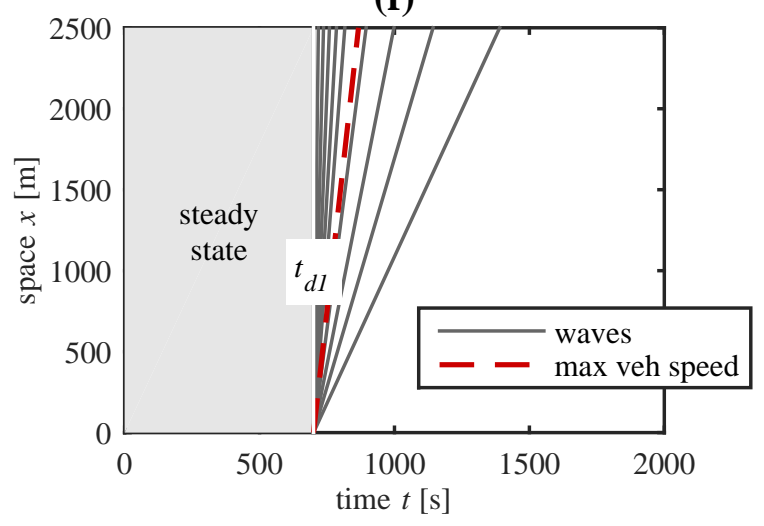

Figure 2: (a) One-staircase demand profile $\lambda(t)$, (b) outflow-MFD with the particular accumulation values $n_{i, 1 \leq i \leq 5}$ indicating the branch intersections and with the levels of demand, (c) evolution of accumulation $n(t)$ and (d) evolution of travel time $T(t)$ where the time $t_{d 1}$ corresponds to a change in demand, and $t_{i 1}$ to a change in MFD branch. (e) Evolution of outflow $\mu(t)$ and (f) representation of wave propagation in the reservoir, a wave being defined as a curve connecting an inflow value at upstream to the same outflow value at downstream. The space axis represents the distance to travel along a trip. Here the maximum vehicle speed is the free-flow speed 
(a)

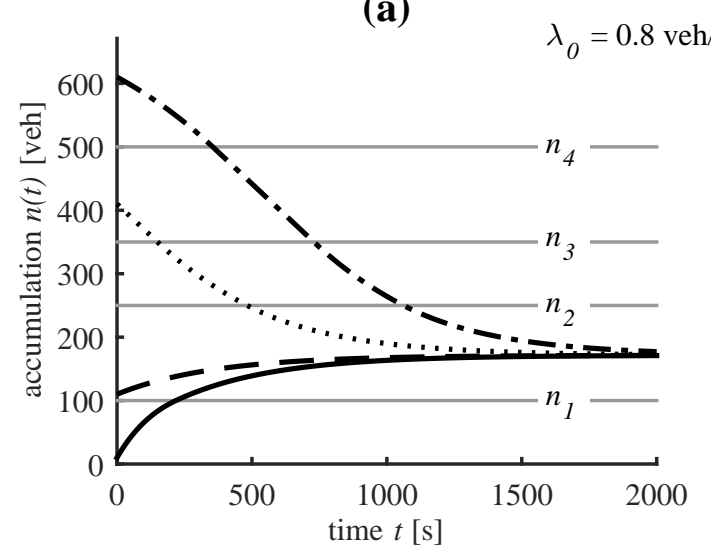

(c)

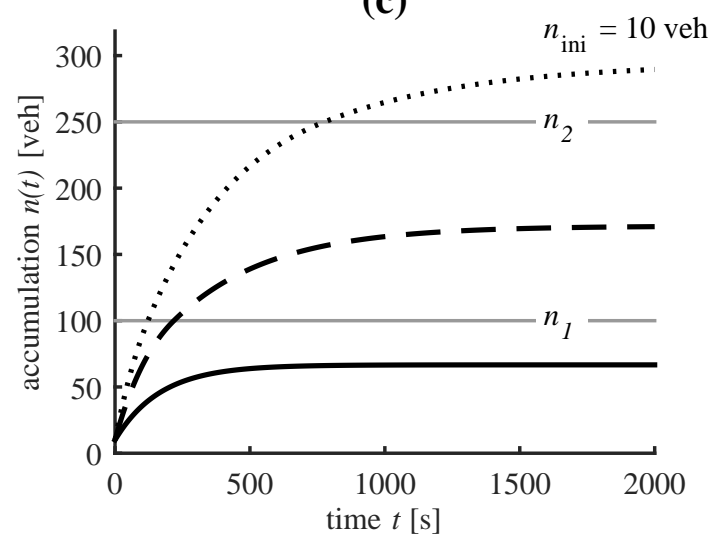

(b)

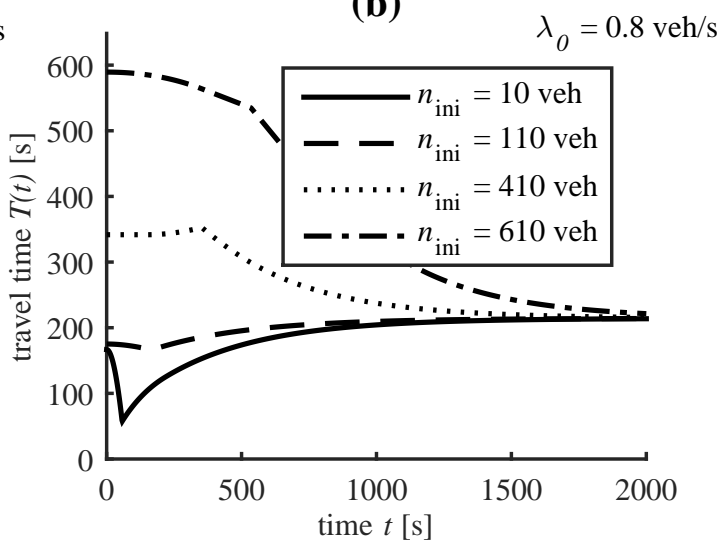

(d)

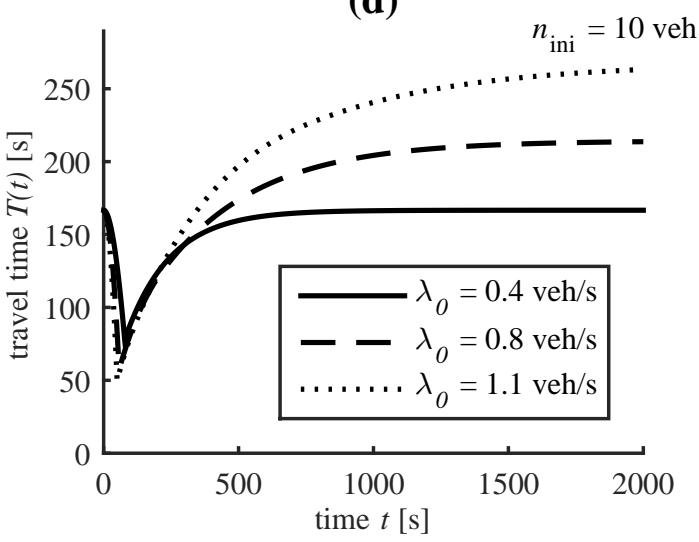

(e)

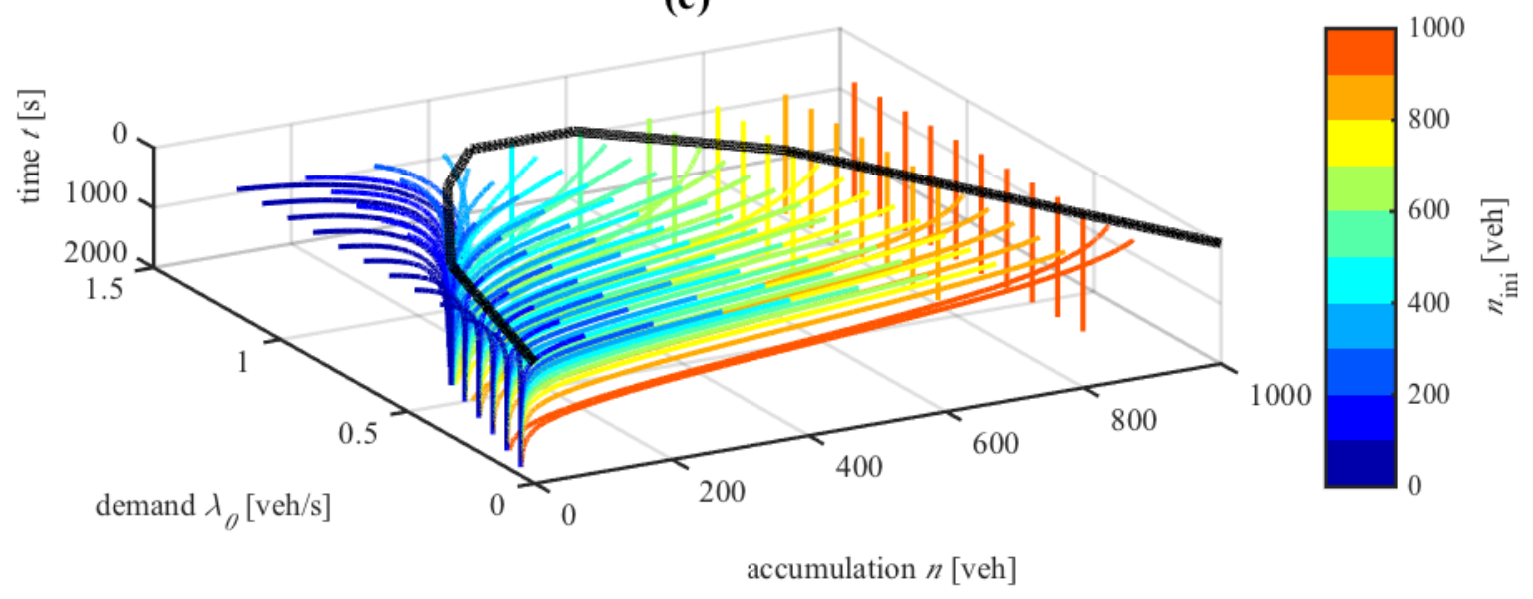

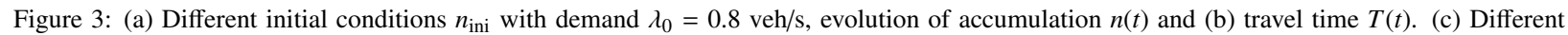
demand levels $\lambda_{0}$ with initial condition $n_{\mathrm{ini}}=10 \mathrm{veh}$, evolution of accumulation $n(t)$ and (d) travel time $T(t)$. (e) Phase diagram in the $\left(\lambda_{0}, n\right.$, $t$ ) space, one line corresponds to the solution with initial conditions $\left(\lambda_{0}, n_{\text {ini }}\right)$, the black line being the outflow-MFD. Initial conditions above the congested part of the MFD lead to steady state solutions with our choice for the reservoir entrance function $I(n)$ 


\subsection{Congestion case and full numerical resolution}

In congestion when the outflow is restricted to a given supply value $\mu_{0}$, equation 2 transforms to $d n / d t=$ $I(n(t))-\mu_{0}$, where $I(n(t))$ is the reservoir inflow. In this case $I(n(t))$ may indeed depend on $n(t)$ because for high values of accumulation the demand is limited by the reservoir entrance function. The expression of $I(n(t))$ is assumed to be equal to the reservoir capacity in free-flow and to correspond to the congested branch of the MFD in congestion (Geroliminis and Daganzo, 2007). This means this differential equation will lead to a quite similar solution as presented in equation 5 in switching the role of inflow and outflow. This shows that our approach is relevant to examine congested situations too, though not presented here. With this shape of $I(n)$, intermediate situations where both inflow and outflow depend on $n(t)$ lead automatically to equilibrium, as $I(n)=O(n)$ on the congested part. Figure 3(e) illustrates the reservoir dynamics in the $\left(\lambda_{0}, n, t\right)$ space. We clearly see that the free-flow part of the outflow-MFD is attractive, whereas the congested part is repulsive: once an initial condition $\left(\lambda_{0}, n_{\text {ini }}\right)$ is below the congested branches the solution will automatically converge to the corresponding point on the free-flow branches. Our choice here for $I(n)$ makes the solutions with $\left(\lambda_{0}, n_{\text {ini }}\right)$ above the congested branches stable and not converging to gridlock, because inflow is instantaneously restricted by $I(n)$ equal to outflow $O(n)$ in congestion.

In order to ease the implementation of the accumulation-based model, a full numerical resolution method can be used. To illustrate, we used a first-order Euler-type finite difference method, where the evolution of accumulation is obtained by the following iterative process:

$$
n(t+\delta t)=n(t)+\delta t \cdot\left(\min [\lambda(t), I(n(t))]-\min \left[\mu_{0}, O(n(t))\right]\right)
$$

where $\delta t$ is the time step, and $\mu_{0}$ (in [veh/s]) is a given supply value at the reservoir exit, which may eventually depend on time. Note that if the inflow is restricted, i.e. $\min [\lambda(t), I(n(t))]=I(n(t))$, the vehicles waiting at the reservoir entry must be taken into account in case of demand evolution. In terms of computational time, the semi-analytical method is about ten times faster than the full numerical one if only a few branches are needed to define the MFD and if the demand scenario has only a few different levels. But the fully numerical method becomes the fastest one in case of any given shape for the MFD and continuous variations of demand. In that case the semi-analytical process will need numerous calculation loops to approximate both the shape of the MFD and the demand evolution. The influence of the number of MFD branches and demand steps for the semi-analytical method, and the time step choice for the full numerical resolution is analyzed in Appendix B. Results show that 8 MFD branches and a 40-step demand profile are sufficient to get accurate approximations of the exact solution. For the full numerical method, a time step equal to $10 \%$ of the reservoir free-flow travel time is small enough to get acceptable numerical errors. The exact solution used to make these comparisons has been obtained in Mathematica ${ }^{\circledR}$, as further discussed in Laval et al. (2017).

\subsection{Validity domain of the accumulation-based model}

Based on the first limitations highlighted in this section for the accumulation-based model, a validity domain can be established regarding the travel time drop phenomenon. Our hypothesis is that this undesirable artifact is due to the demand curve varying too fast. Daganzo (2007) already conjectured that the accumulation-based model is suitable for slow varying boundary conditions only. What we propose here is to characterize the demand change rate threshold below which the model can be considered accurate. For this purpose we perform numerous simulations with a logistic "S-shaped" demand defined by two parameters: the demand gap $\Delta \lambda$ and the gap duration $\Delta t$. The demand variation rate is described by $Q_{\lambda}=\Delta \lambda / \Delta t$. We observe then the evolution of the travel time drop height $h_{0}$ and its duration $d_{0}$ as presented in figures 4(a) and (b). If we compare the blue curve $(\Delta t=0.2 \mathrm{~s})$ with the yellow one $(\Delta t=800 \mathrm{~s})$, we see that the faster the demand varies, the higher the travel time drop $h_{0}$ is, but the shorter its duration $d_{0}$ is. Therefore we could expect $h_{0}$ and $d_{0}$ evolving in opposite directions as $Q_{\lambda}$ increases. That is exactly what figures 4(c) and (d) show, where $h_{0}$ and $d_{0}$ are plotted in the $(\Delta t, \Delta \lambda)$ plane. White lines represent the iso- $Q_{\lambda}$ curves. Both effects of the drop height and drop duration can be visualized in figure 4(e) where the product $h_{0} \cdot d_{0}$ is plotted.

These graphs point out that the relationship between the travel time drop phenomenon and the demand variation rate is not obvious, and only considering the value of $Q_{\lambda}$ to define an acceptable validity domain of the model may be too restrictive. Considering the global effect, i.e. the product $h_{0} \cdot d_{0}$, it seems that the demand gap $\Delta \lambda$ alone is relevant for the characterization of the validity domain. For example we could claim that a demand increase below $0.1 \mathrm{veh} / \mathrm{s}$ ensures the accumulation-based model accuracy in travel time description, whatever the time it takes. However, using only the indicator $h_{0} \cdot d_{0}$ makes these two situations equivalent: $h_{0}=60 \mathrm{~s}$ during $d_{0}=1000 \mathrm{~s}$, and $h_{0}=6 \mathrm{~s}$ during 
$d_{0}=10,000 \mathrm{~s}$, which may not be appropriate for cases when very small differences in travel time are considered negligible. So if we suppose the drop height is more prejudicial than its duration, only the indicator $h_{0}$ should be used. In that case, the validity domain would be $\Delta \lambda<0.1 \mathrm{veh} / \mathrm{s}$ or $Q_{\lambda}<10^{-4} \mathrm{veh} / \mathrm{s}^{2}$. Note that these values are completely dependent on our numerical application. An attempt to provide a validity domain applicable for any single reservoir problem is presented in Appendix B. 
(a)

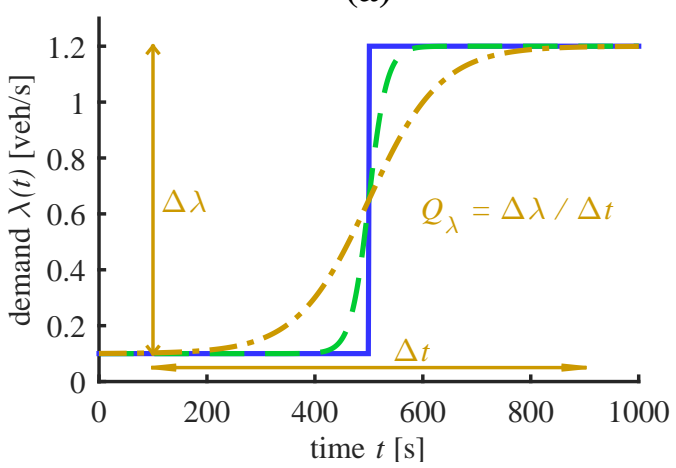

(b)

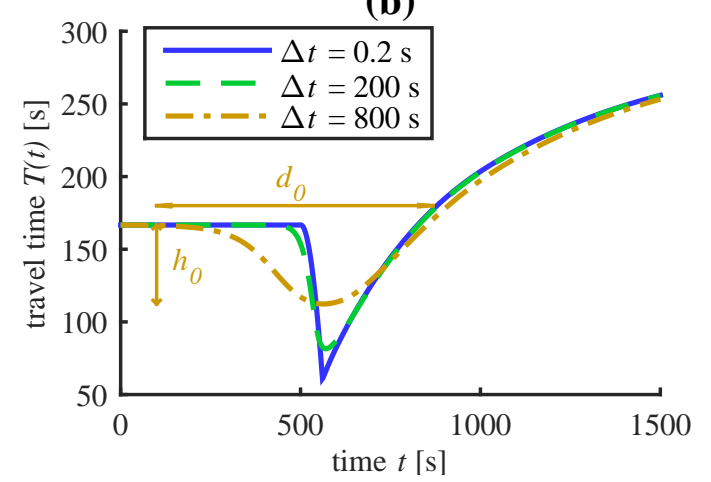

(c)

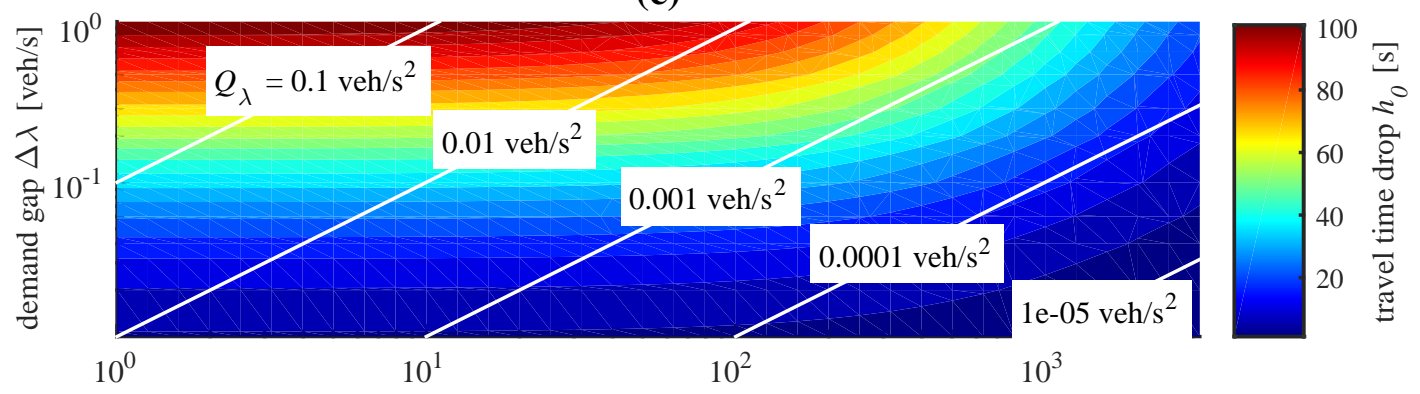

(d)

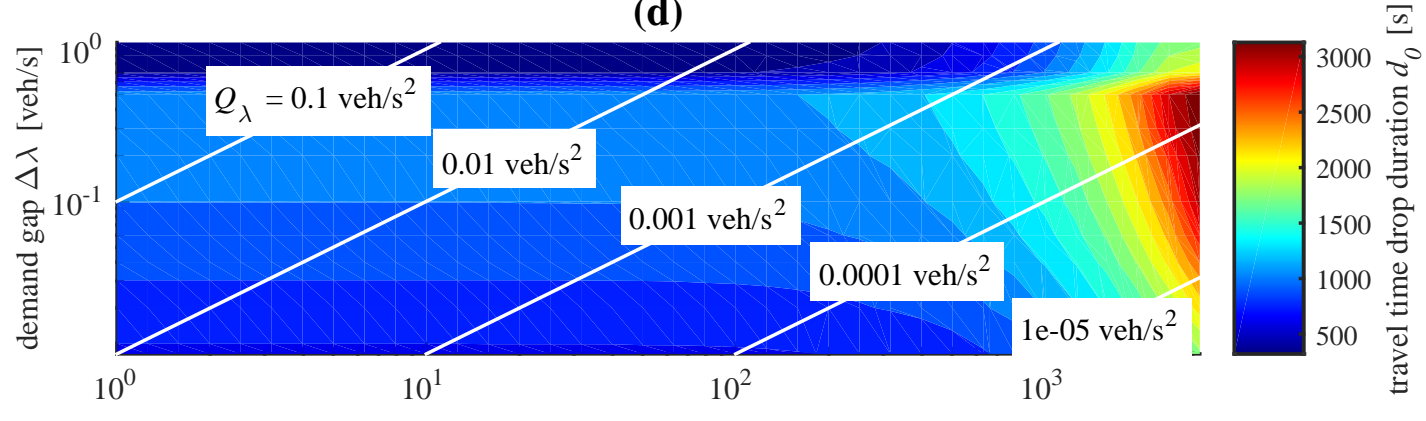

(e)

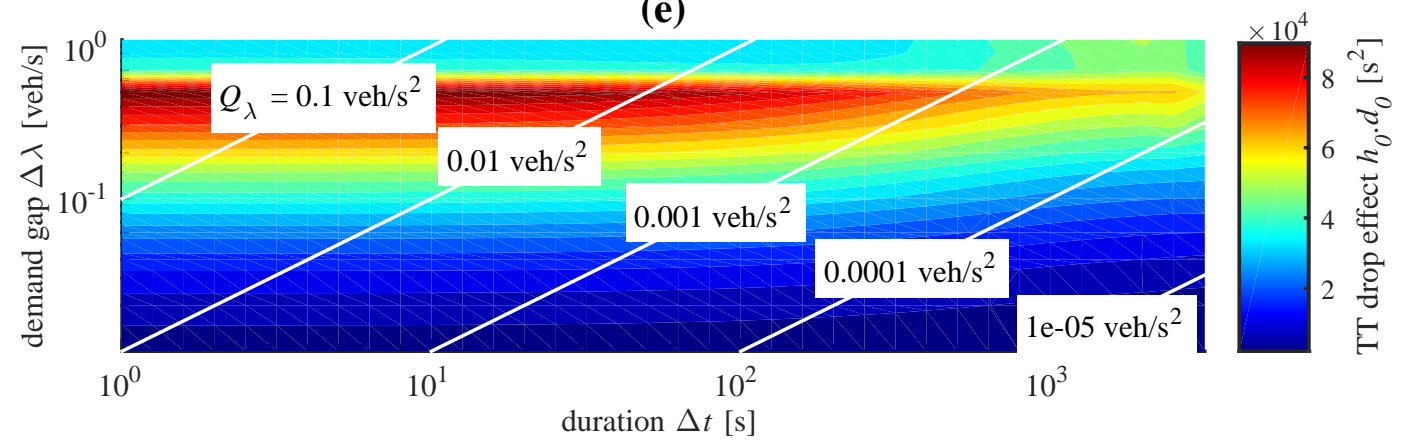

Figure 4: Influence of the demand variation rate on the accumulation-based model accuracy for travel time. This accuracy is defined regarding the size of the travel time drop artifact. (a) Demand profiles with different variation rates, (b) resulting travel time profiles where two inaccuracy factors are measured: the drop height $h_{0}$ and the drop duration $d_{0}$, (c) drop height value in the ( $\left.\Delta t, \Delta \lambda\right)$ plane, (d) drop duration value, and (e) the product height-duration value in the same plane 


\section{Trip-based approach}

\subsection{Theoretical basis}

The general principle of the trip-based approach is to derive the inflow and outflow curves noting that the travel distance $L$ by a driver entering at time $t-T(t)$ should satisfy:

$$
L=\int_{t-T(t)}^{t} V(n(s)) d s
$$

This definition is mentioned in the $11^{\text {th }}$ footnote of Arnott (2013) first, and used in the studies of Fosgerau (2015) and Lamotte and Geroliminis (2016) then. Daganzo and Lehe (2015) also made use of such an approach, but without explicitly mentioning equation 8 . Note that equation 8 also resort on the notion of MFD to define the common speed of vehicles within the reservoir at time $t$. Note also that this formulation focuses on the distance $L$ traveled by the vehicle entering at $t-T(t)$ and exiting at $T(t)$, which is defined as the integration of the reservoir speed during the vehicle traveling period. The evolution of the accumulation $n(t)$ is then only defined in an implicit way, i.e. it results from vehicles entering and exiting the reservoir. With this formulation of the reservoir system, it appears clearly that the definition of the individual trip length is no longer a problem as it becomes part of the input parameters. In this section, we will first consider that $L$ is the same for all travelers. This assumption allows us to develop a proper resolution scheme, and ensures the validity of the comparisons we will make with the accumulation-based model. This hypothesis will be relaxed in section 3.4 when presenting our event-based numerical resolution method.

To see the fundamental difference between the trip- and the accumulation-based models, we rewrite the definition of $L$ for the latter. If we use the formulation of the travel time based on outflow, namely $n(t-T(t))=N_{\text {out }}(t)-N_{\text {out }}(t-$ $T(t))=\int_{t-T(t)}^{t} O(n(s)) d s$, and combine it with equation 1 we find: $n(t-T(t))=\int_{t-T(t)}^{t} \frac{P(n(s))}{L} d s$. As a consequence, we get the following expression for $L$ in the accumulation-based model framework:

$$
L=\frac{1}{n(t-T(t))} \int_{t-T(t)}^{t} P(n(s)) d s \quad \text { (accumulation-based) }
$$

This has to be compared with equation 8 for the trip-based model, in which $V(n(s))$ is replaced by $P(n(s)) / n(s)$ :

$$
L=\int_{t-T(t)}^{t} \frac{P(n(s))}{n(s)} d s \quad \text { (trip-based) }
$$

It becomes apparent that the accumulation-based model considers the accumulation $n(s)(s \in[t-T(t), t])$ constant and equal to $n(t-T(t))$ during the travel duration $[t-T(t), t]$ of the vehicle exiting at $t$ for the trip length calculation. On the other hand, the trip-based model takes the accumulation evolution into account during the same period, which is more realistic. In particular, this comparison shows that the two approaches are equivalent in steady state, or when the accumulation varies slowly which is consistent with slow demand variations. In other cases, e.g. as in peak-hour periods, we expect the accumulation-based model to be inaccurate during transition phases.

While being the first to introduce the trip-based model formulation, Arnott (2013) mentioned that its analytical resolution is almost impossible considering the current advances in mathematics. So, he adopted the accumulationbased framework in the following of his paper. Fosgerau (2015) proposed some analytical developments of equation 8 but defined the departure time rate as a function of the trip length (two users sharing the same trip distance have necessarily the same departure time), which seems too restrictive for us. Daganzo and Lehe (2015) and then Lamotte and Geroliminis (2016) presented an interesting resolution method for the trip-based model that consists in assigning to each user a trip length and let him/her travels at the global mean speed $V(n(t))$ until he/she completes his/her trip and exits the reservoir. As the departure rate is considered as an input of the simulation, this method provides the arrival rate, i.e. the outflow of the system. Here, we propose an elegant way to get numerical solutions from the trip length equation 8 while controlling the accuracy of the approximation by using Taylor developments. This confirms that the methods originally proposed by Daganzo and Lehe (2015) and Lamotte and Geroliminis (2016) provide accurate results when discretizing the entry flow in small vehicle fractions. Our numerical approximation is presented in the following sections 3.2 and 3.3. Finally, it appears that Daganzo and Lehe (2015) are using a time step to perform the simulation. We will show in section 3.4 that like in Lamotte and Geroliminis (2016) we can get rid of time steps to achieve a full event-based simulation process when discretizing the entry flow in units of vehicles. 


\subsection{Numerical approximation}

In a first time, we solve the problem under free-flow conditions. The congested case will be studied latter. In a free-flow situation, i.e. when inflow and outflow are not restricted, the behavior of the system is governed by the demand and solving the problem consists in determining the outflow $\mu(t)$. Here the basic idea is to use equation 8 to express the latter. From the definition of the experienced travel time $T(t)$ we know that $N_{\text {out }}(t)=N_{\text {in }}(t-T(t))$ for the cumulative count curves $N_{\text {in }}(t)$ and $N_{\text {out }}(t)$, see figure 1(b). The derivative of $N_{\text {out }}(t)$ with respect to time leads to: $\mu(t)=(1-d T / d t) \lambda(t-T(t))$. On the other hand, as $L$ is considered constant here, the time derivative of equation 8 is: $0=V(n(t))-(1-d T / d t) V(n(t-T(t)))$. Combining these two relationships gives an explicit expression of $\mu(t)$ :

$$
\mu(t)=\lambda(t-T(t)) \cdot \frac{V(n(t))}{V(n(t-T(t)))}
$$

This result has already been mentioned by Arnott (2013) as an equivalent problem to equation 8. Introducing this expression into the state equation 2 still remains an intractable mathematical problem because it corresponds to a differential equation with endogenous delay. However this expression can be transformed into an efficient numerical approximation by discretizing vehicles into fractions $\delta N$.

Let us assume that all the reservoir states are known until a given time $t_{1}$ : the demand $\lambda(t)$, the outflow $\mu(t)$, the accumulation $n(t)$ and the travel time $T(t)$. We then want to solve the reservoir dynamics for $t>t_{1}$. The vehicle exiting at $t_{1}$ is denoted by the continuous index $N$, its entering and exiting times are written $t_{N}^{\text {in }}=t_{0}$ and $t_{N}^{\text {out }}=t_{1}$ respectively. The following vehicle is denoted by the index $N+\delta N$ and its entering and exiting times are $t_{N+\delta N}^{\text {in }}$ and $t_{N+\delta N}^{\text {out }}$. Physically speaking $\delta N$ should be equal to 1 and represents one vehicle. However, we deal here with a continuous model, so we can fix $\delta N$ to very small values to investigate numerical accuracy. Since we are in free-flow conditions the entering vehicle time is determined by the demand, thus we have also $t_{N+\delta N}^{\text {in }}=t_{N}^{\text {in }}+\delta N / \lambda\left(t_{N}^{\text {in }}\right)$. Solving the problem for this vehicle is to determine its exit time $t_{N+\delta N}^{\text {out }}=t_{1}+\delta t$, that is to say, to determine the time increment $\delta t$ after the last exit, see figure 5(a). With these notations equation 11 can be approximated by:

$$
\frac{\delta N}{\delta t}=\lambda\left(t_{N}^{\text {in }}\right) \cdot \frac{V\left(n\left(t_{N}^{\text {out }}\right)\right)}{V\left(n\left(t_{N}^{\text {in }}\right)\right)}
$$

which gives directly an estimation for $\delta t$. Actually this corresponds to a first-order approximation on the exiting vehicle curve as the slope $\mu(t)$ is estimated by $\delta N / \delta t$. However we have found an interesting way to perform the calculation of $\delta t$ at higher orders. This method is detailed in Appendix A, results show that in fact the estimation of $\delta t$ at first order is largely sufficient if $\delta N$ is small enough (around $0.1 \mathrm{veh}$ ).
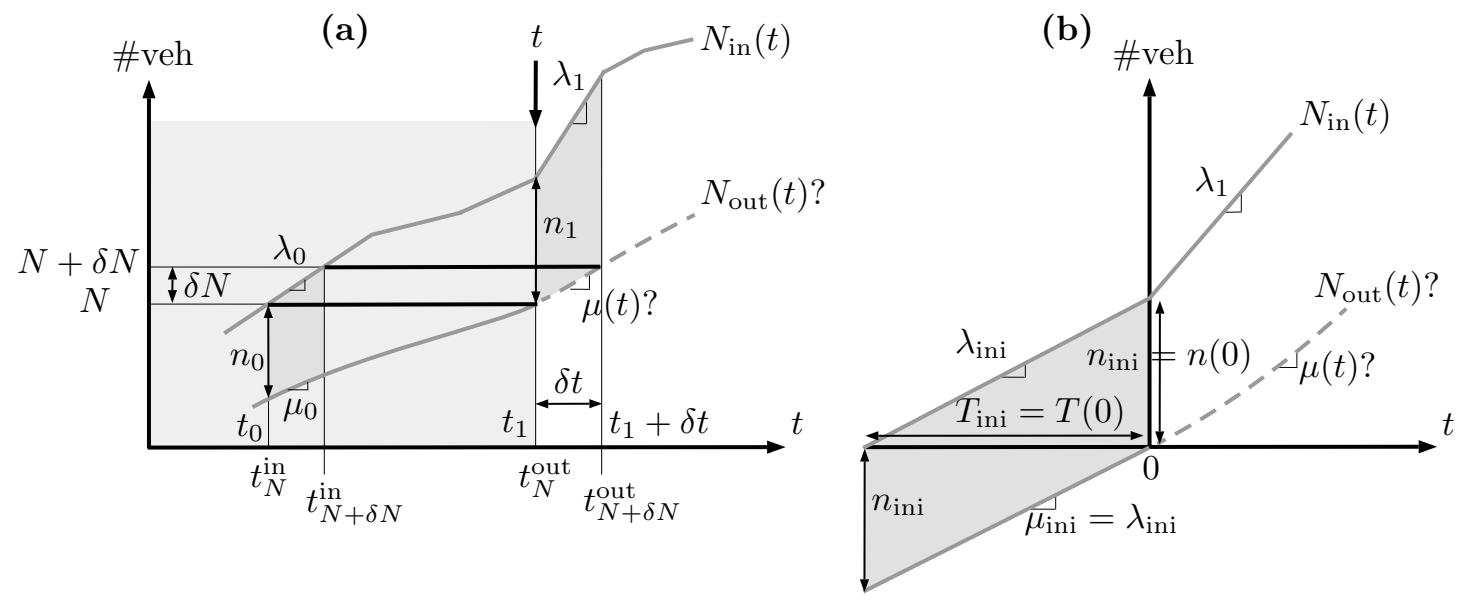

Figure 5: (a) Representation of our resolution scheme on the cumulative count curves, the system evolution is supposed known until $t_{1}=t_{N}^{\text {out }},($ b) explanation for the first step of the resolution process, the system is supposed to be in a steady state before $t=0$ determined by the choice of initial accumulation $n_{\text {ini }}$ 


\subsection{Step-by-step numerical resolution in the general case}

We present now the complete algorithm to solve any problem that complies with our study hypotheses stated in section 2. It is based on the method previously described and summarized in figure 5(a). Given a choice of a vehicle increment $\delta N$, we estimate the exiting vehicle curve by calculating the time step $\delta t$ after which the next vehicle $N+\delta N$ will exit. Since all the travelers have the same trip length $L$, this method is consistent with the First-In, FirstOut (FIFO) rule which ensures the validity of this process. Note that unlike the accumulation-based model which has no memory, solving the system dynamics at any time $t_{1}$ for the trip-based model requires the knowledge of the past of the system. This may be highlighted as another fundamental difference between the two modeling approaches. In practice, this will be a problem for the simulation initialization, when the first vehicles start to exit the reservoir without knowing their entering times. This issue is tackled by simply considering that the system is in a steady state completely defined by the choice of the initial accumulation $n_{\text {ini }}$ for $t<0$. This is illustrated in figure 5(b), the demand in the steady state before $t=0$ is given by $\lambda_{\text {ini }}=n_{\text {ini }} V\left(n_{\text {ini }}\right) / L$. In fact, the same consideration was also observed with the accumulation-based model to define properly the travel time evolution during the warm-up period. Hence for the simulation initialization the two modeling approaches are consistent with each other.

The numerical resolution process of the trip-based model is then summarized below:

1. Choose a vehicle increment $\delta N$

2. Initialize $t_{1}=0, n_{1}=n_{\mathrm{ini}}, V_{1}=V\left(n_{1}\right), T_{1}=L / V_{1}$ and for the past of the system $t_{0}=-T_{1}, n_{0}=n_{1}, V_{0}=V_{1}$, $\lambda_{0}=n_{0} V_{0} / L, \mu_{0}=\lambda_{0}$

3. Save the current level of demand $\lambda_{1}$

4. if $t_{0}>0$, update at $t=t_{0}: n_{0}=n\left(t_{0}\right), V_{0}=V\left(n_{0}\right), \lambda_{0}=\lambda\left(t_{0}\right), \mu_{0}=\mu\left(t_{0}\right)$

5. Determine the current branch of the production-MFD $\left(w_{i}, \eta_{i}\right)$

6. Update the mean speed $V_{1}=V\left(n_{1}\right)$

7. Calculate the next time step $\delta t$ at the chosen order, using equation A.6 for order 1 or equation A.7 for order 2

8. Update at $t=t_{1}: t_{1}=t_{1}+\delta t, N_{\text {in }}=N_{\text {in }}+\lambda_{1} \delta t, N_{\text {out }}=N_{\text {out }}+\delta N, \mu=\delta N / \delta t, n_{1}=N_{\text {in }}-N_{\text {out }}, t_{0}=t_{0}+\delta N / \lambda_{0}$, $T_{1}=t_{1}-t_{0}$

9. If $t_{1}$ has not reached the next change in demand yet, go to 4 . Else go to 3 .

The application of this process is illustrated on the simple one-staircase demand profile that we used in section 2.4. The calculation of $\delta t$ is computed at order 1 with $\delta N=0.1$ veh. Figures 6(a) and 7(b) show the accumulation and travel time evolution in solid line, the dashed lines represent the previous solutions obtained with the accumulationbased model. This example immediately points out the benefit of the trip-based approach, as the results for the travel time are now consistent. In this case, the absence of drop in travel time may be explained by the fact that the tripbased model keeps the memory of the past of the system, and thus provides more reliable results when the boundary conditions are changing. Moreover we notice that this model is more reactive than the accumulation-based one, the latter suffers indeed from intrinsic viscosity phenomenon due to the form of equation 4. Figure 6(d) shows the wave pattern associated to the trip-based model. This pattern has been constructed similarly as the one for the accumulationbased model in figure 2(f). It appears that now no wave is traveling faster than the vehicles, and thus that travel times are properly reproduced by the trip-based approach. Finally, it should be mentioned that the trip-based model may exhibit local inconsistencies with respect to the outflow curve, see figure 6(c). When the demand surge is observed at time $t_{d 1}$ the outflow is constant as expected, but then slightly decreases before the surge in outflow is observed. The reason is that the trip-based model, while providing a much better description of vehicle trips, still resorts to an homogeneous speed for all the vehicles within the reservoir. Therefore, even the vehicles that enter the reservoir before the demand surge are slow down by the increase of accumulation resulting from this event.

Figure 7 presents, similarly as in figure 3 , the evolution of accumulation and travel time for different initial conditions $n_{\text {ini }}$ and different levels of demand $\lambda_{0}$. Again because $\lambda_{0} \neq \lambda_{\text {ini }}$, the system enters the transient phase at $t=0$. The same conclusions are observed, namely the trip-based model gives more realistic travel times in transient phases, and is also more reactive than the accumulation-based one. In particular we can notice the very bad description of $T(t)$ in the accumulation-based framework compared with the trip-based one when the gap of demand is high. For example in figures 7(b) or (d) for $n_{\mathrm{ini}}=10 \mathrm{veh}$ (which corresponds to $\lambda_{\mathrm{ini}}=0.06 \mathrm{veh} / \mathrm{s}$ ), and for $\lambda_{0}=0.8 \mathrm{veh} / \mathrm{s}$, the travel time described by the accumulation-based model exhibits a high drop during the whole transition period of the travel time provided by the trip-based model. On the other hand when the value of $\lambda_{0}$ is close to the initial state $\lambda_{\text {ini }}$, the effect 
(a)

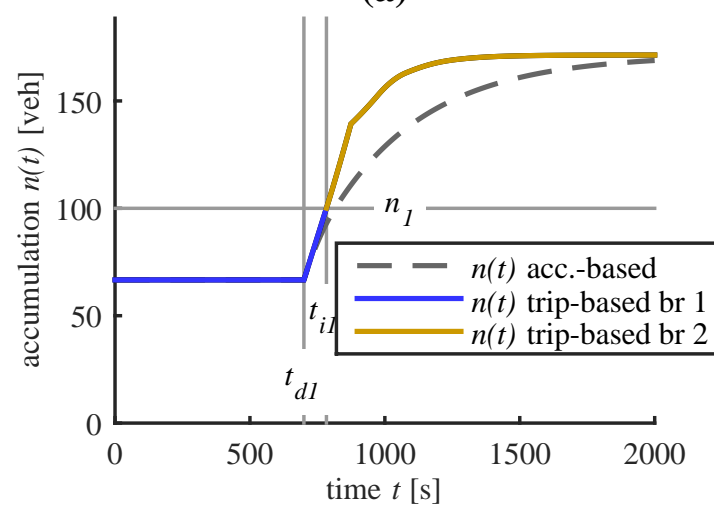

(c)

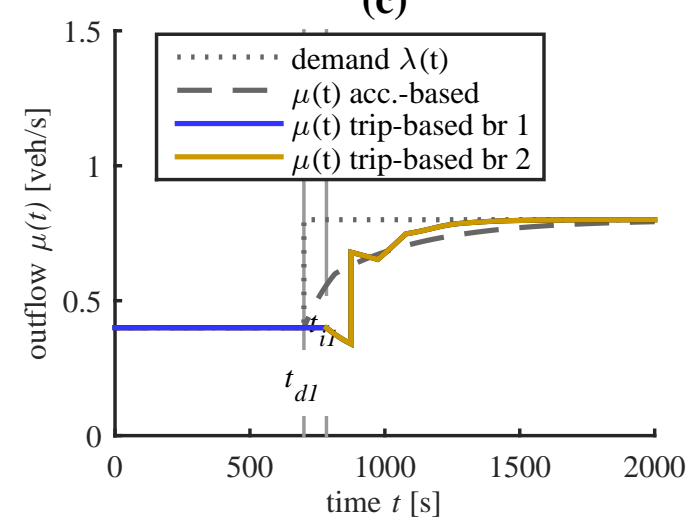

(e)

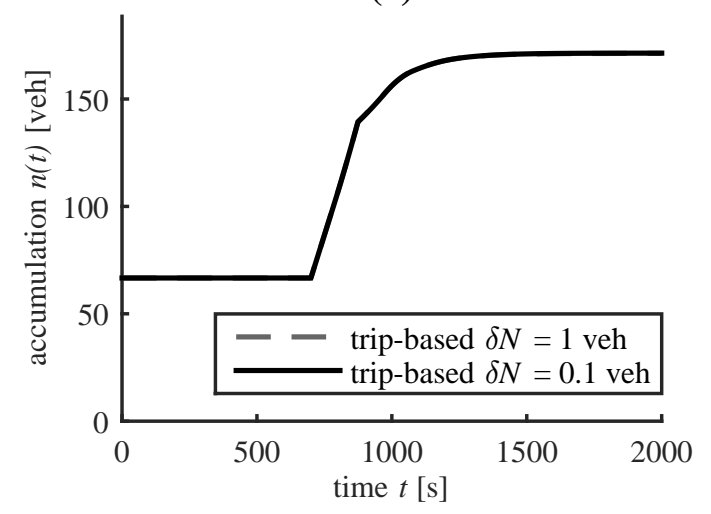

(b)

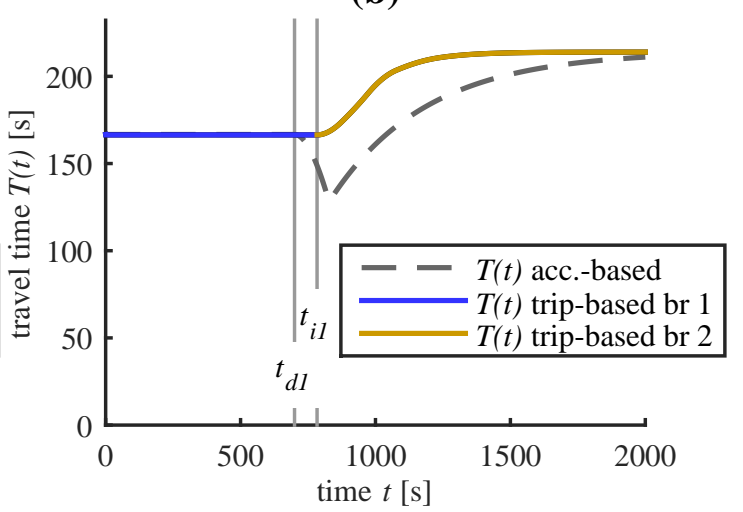

(d)

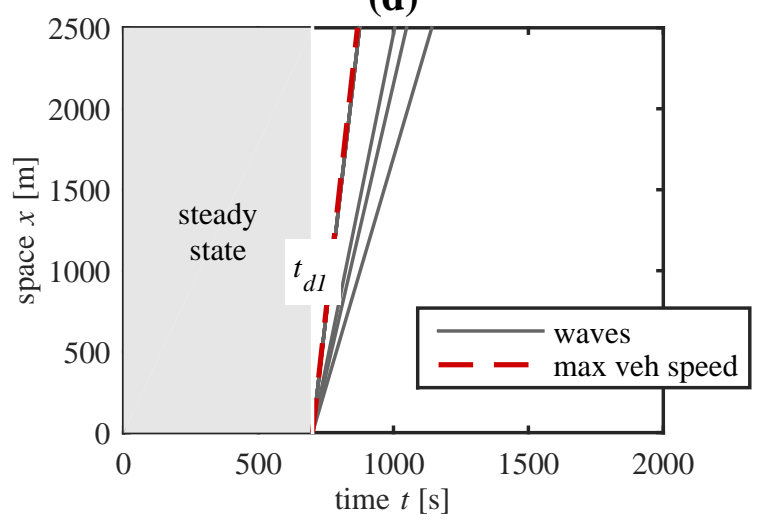

(f)

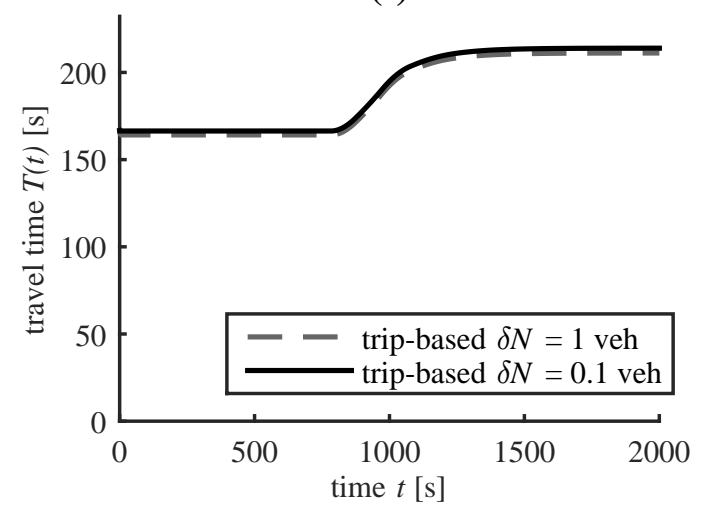

Figure 6: Comparison between accumulation-based and trip-based models, the latter is performed with $\delta N=0.1$ veh. (a) Evolution of accumulation $n(t)$ and (b) evolution of travel time $T(t)$ where the time $t_{d 1}$ corresponds to a change in demand, and $t_{i 1}$ to a change in MFD branch, also indicated by the change in color ("br" stands for "branch"). (c) Evolution of outflow $\mu(t)$ and (d) representation of wave propagation in the reservoir for the trip-based model, a wave being defined as a curve connecting an inflow value at upstream to the same outflow value at downstream. The maximum vehicle speed is the free-flow speed. (e) Comparison for the trip-based model with two values of $\delta N$, evolution of accumulation $n(t)$ and (f) evolution of travel time $T(t)$

of the travel time drop is minimized. An example is the case $n_{\mathrm{ini}}=110 \mathrm{veh}$ (which corresponds to $\lambda_{\mathrm{ini}}=0.66 \mathrm{veh} / \mathrm{s}$ ) 
with $\lambda_{0}=0.8 \mathrm{veh} / \mathrm{s}$ in figure 7(b). In this situation the solutions for $T(t)$ are quite similar in both approaches. This corroborates our analysis on the validity domain of the accumulation-based model in section 2.6, for which we notice that the height of the demand gap $\Delta \lambda$ seem to have the most influence on the travel time drop, in comparison with the gap duration $\Delta t$.

(a)

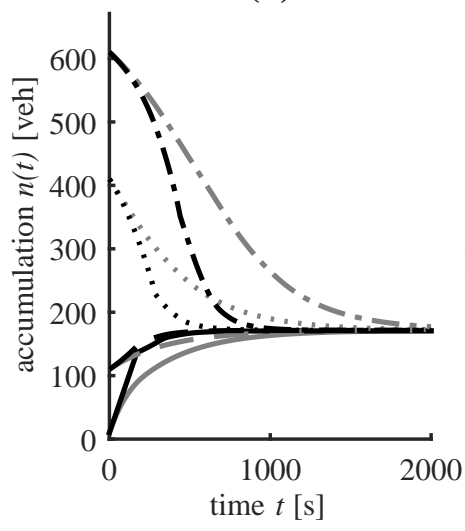

(c)

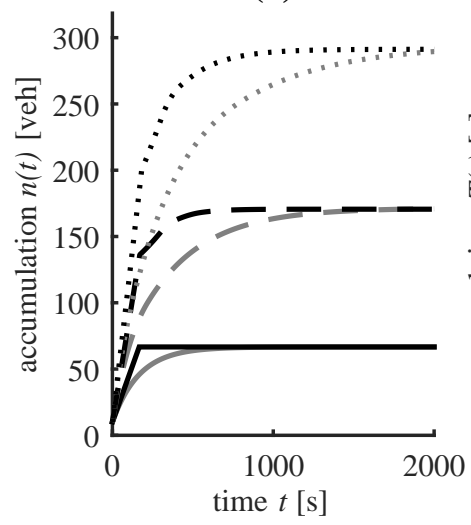

(b)

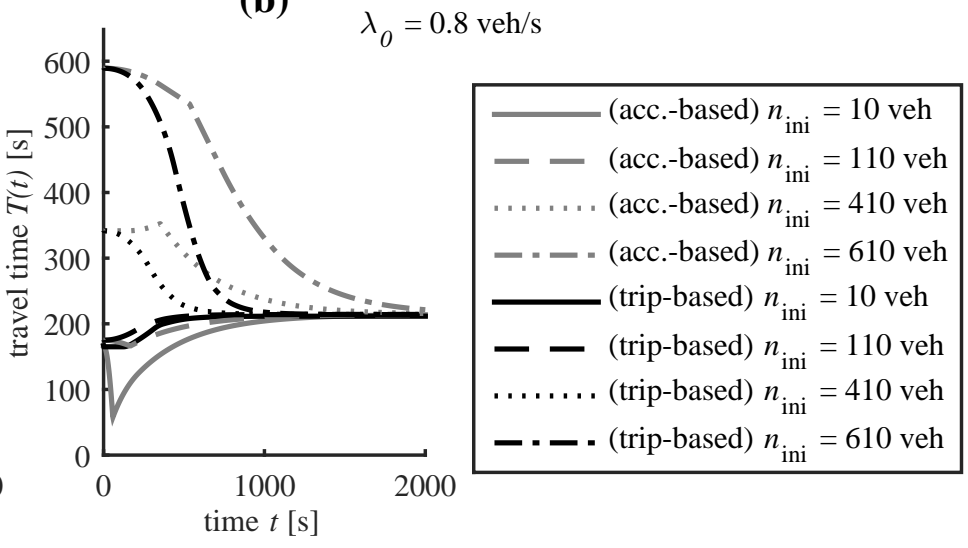

(d)

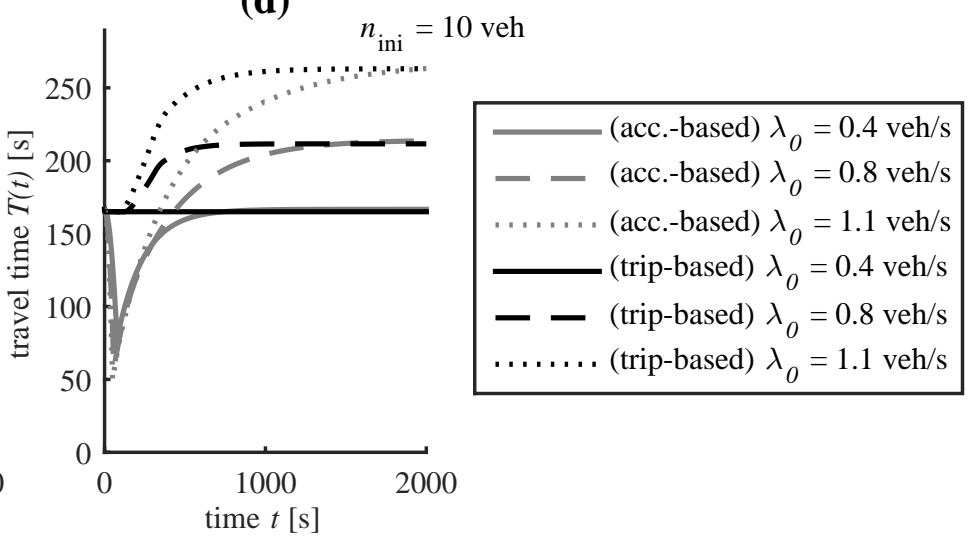

Figure 7: Comparison between accumulation-based and trip-based models. (a) Different initial conditions $n_{\text {ini }}$ with demand $\lambda_{0}=0.8$ veh/s, evolution of accumulation $n(t)$ and (b) travel time $T(t)$. (c) Different demand levels $\lambda_{0}$ with initial condition $n_{\text {ini }}=10$ veh, evolution of accumulation $n(t)$ and (d) travel time $T(t)$

\subsection{The event-based resolution method}

This solver uses the same discretization of entering vehicles into vehicle units like in Daganzo and Lehe (2015) and Lamotte and Geroliminis (2016): we create the vehicles entering the system in accordance with a given demand level, and assign them a chosen trip length, which may be eventually different for each vehicle. The users travel at the current global mean speed $V(n(t))$ given by the speed-MFD of the reservoir for the current accumulation $n(t)$, and exit the reservoir once they complete their assigned trip length. This principle is illustrated in figure 8 . Like in Lamotte and Geroliminis (2016), our event-based simulator does not update the system on a regular time basis, but each time a vehicle enters or exits the network. Our method takes advantage of the fact that the mean speed is the same for all travelers, so that the update of $V(n)$ is only needed when the accumulation $n$ changes, i.e. at a vehicle entry or exit. This speeds up computation as the resulting method is fully event-based without any variable update on a fixed basis (no need for time step). Our resolution scheme adapts by itself to the evolution of the system and is 
always numerically accurate by construction. When the trip length is the same for all users this approach corresponds precisely to the discretization of the trip-based model, and is similar to the previous resolution method computed at order 1 with $\delta N=1$. Comparisons between $\delta N=0.1$ and $\delta N=1$ for the previous method show similar results in figures $6(\mathrm{c})$ and (d).

The main structure of the event-based method is detailed below:

1. Entry of the initial vehicles in the reservoir with the same trip lengths. Like the previous resolution methods, a steady state characterized by this initial accumulation is considered before $t=0$. The traveled distance of these vehicles is then updated at $t=0$ in accordance with this hypothesis.

2. Determine the next event which is the minimum between the next vehicle entry (according to the current demand level), and the next vehicle exit. This gives the next time step $\delta t$.

3. Update the traveled distance for all the circulating vehicles—i.e. which have not finished their trip yet—during $\delta t$ using the mean speed $V(n)$. Actually this update can be done one for all for the next exiting vehicles by storing the speed evolution, because all the users travel at the same speed within the reservoir. This can speed up calculations.

4. If the next event is a vehicle entry, create a new vehicle in the reservoir and assign it a given trip length. Else if it is a vehicle exit, remove the corresponding vehicle from the reservoir. Update the accumulation $n$.

5. Update the speed of all the circulating vehicles, evaluated at $V(n)$.

6. Go to 2 .

The procedure stops when the simulation time reaches a given simulation duration.

In closing, it is important to point out that under congested conditions the two resolution schemes proposed for the trip-based model apply with minimal changes. We propose a three-step procedure, where (i) the problem is solved assuming no supply restriction, (ii) the outflow is reduced according to the supply profile, and (iii) Newell's minimum principle (Newell, 1993) is applied to take the congestion wave impact on inflow into account.

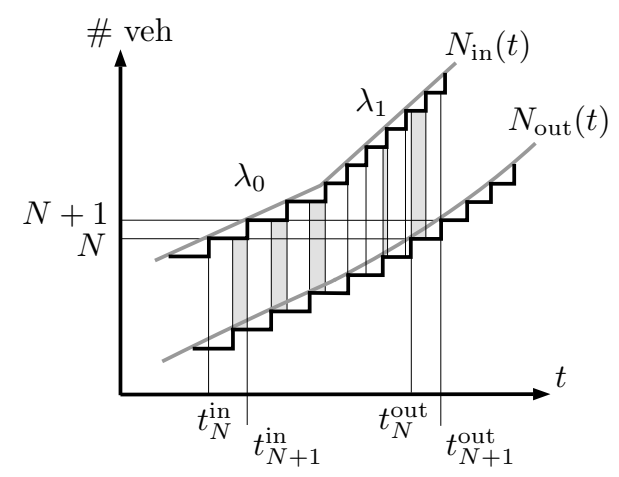

Figure 8: Representation of the event-based resolution method in case of a unique trip length for all travelers (FIFO process) 


\section{Comparison between the accumulation-based and the trip-based models on three case studies}

\subsection{Typical peak-hour demand profile}

We apply now the event-based method on a peak-hour demand case. We compare the results with the resolution scheme of the trip-based model established in the previous section and the accumulation-based model. Two simulations are performed: the first one is considering a slow-varying demand with a simulation duration of $10,000 \mathrm{~s}$, whereas the second one is characterized by much faster demand variations as the whole simulation lasts only $2000 \mathrm{~s}$. The two demand profiles are shown in figures 9(a) and 9(e), they are identical in shape and reach the same levels. The continuous aspect of these profiles is actually approximated by successive small stairs to comply with the piecewise constant hypothesis.

Figures 9(b)-(d) present the results for the first simulation, and figures 9(f)-(h) the results for the second simulation. It appears clearly that the accumulation-based model is more suitable for slow-varying demand situations, as already mentioned in Daganzo (2007). In case of fast variations in demand, the slow reaction time of this model, already highlighted in section 3, makes it underestimate the accumulation peak in figure 9(f). The drop phenomenon in travel time is also noticed in both simulations, but the estimation of $T(t)$ is clearly worse in the second case. For the latter the drop size could have been forecasted thanks to our study in section 2.6. Given an increase of 1 veh/s over $500 \mathrm{~s}$ for the demand brings us to a drop of $90 \mathrm{~s}$ during $500 \mathrm{~s}$. This is indeed what is observed in figure $9(\mathrm{~g})$, although the drop height is a bit overestimated. Another phenomenon worth mentioning is the counter-clockwise hysteresis loop occurring for the trip-based model when displaying the traffic states in the (accumulation, outflow) plane, see figures 9(d) and 9(h). The same phenomenon has been also observed by Lamotte and Geroliminis (2016). On the other hand, experimental (e.g. Buisson and Ladier, 2009; Geroliminis and Sun, 2011), theoretical (e.g. Gayah and Daganzo, 2011) and simulation (e.g. Mahmassani et al., 2013) studies suggested that clockwise hysteresis loops are more likely to occur in the production-MFD. These authors gave useful insights on heterogeneity in traffic density being one of the causes of hysteresis loops. For instance, Gayah and Daganzo (2011) highlighted hysteresis in a simplistic two-bin system due to density heterogeneity between the two bins, their modeling approach being grounded on the accumulation-based model. Some authors like Daganzo (2011) explained these phenomena by a growing effect of instabilities in the network responsible for internal capacity drops during the recovery of congestion. In our framework this effect is not modeled, nor potential internal heterogeneity in density. The counter-clockwise loop we observe in the trip-based approach has a completely different explanation, and to the authors' best knowledge, this has never been exhibited in previous works on hysteresis in MFD, on the notable exception of Lamotte and Geroliminis (2016). This phenomenon is a direct consequence of the delay between the evolution of accumulation and the one of outflow which is reproduced by the trip-based model. This generates traffic states which are below the outflow-MFD during the onset of congestion (accumulation varies faster than outflow due to the time needed for inflow information to travel from upstream to downstream), and then traffic states that are above the outflow-MFD during the offset of congestion (the reduction of accumulation is faster than the one of outflow for the same reason). It should be also noticed that our observations apply to the outflow-MFD and not to the production-MFD. This is another major difference compared with the above-mentioned studies, which either make observation on the production-MFD or consider that outflowand production-MFD are simply proportional (use of the accumulation-based model). More data and especially reliable outflow data are required to have a complete picture of which effects are the most significant. All effects can also be combined for real observations. 
(a)

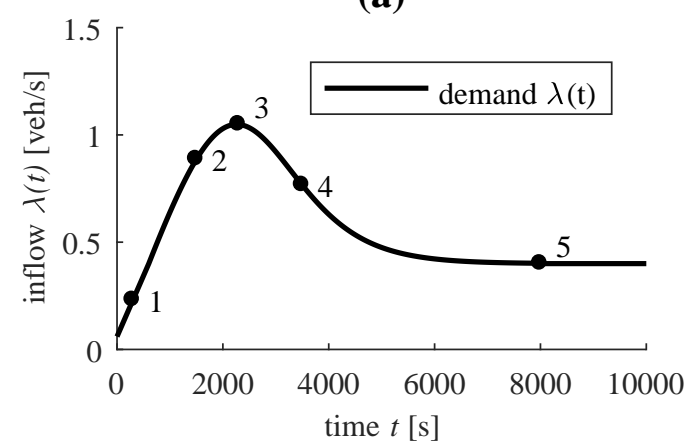

(c)

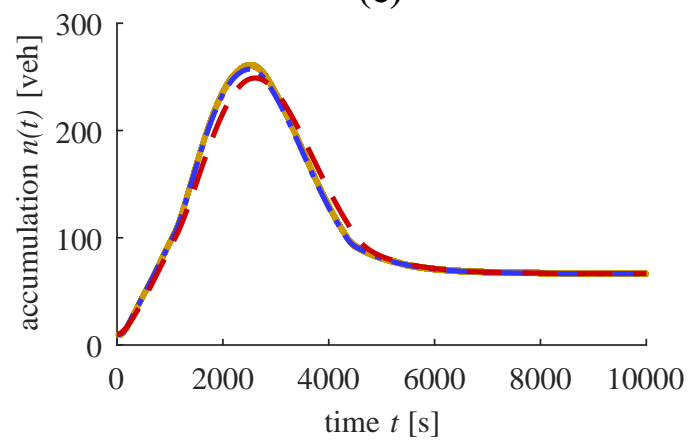

(e)

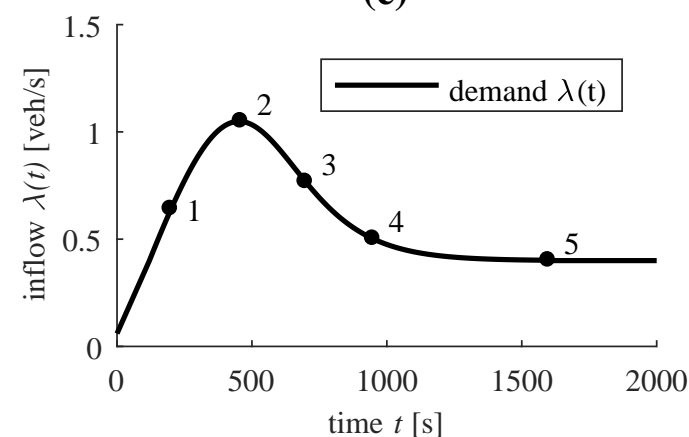

(g)

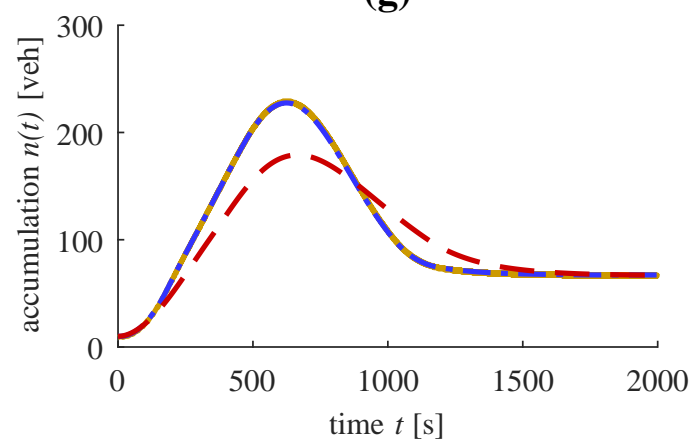

(b)

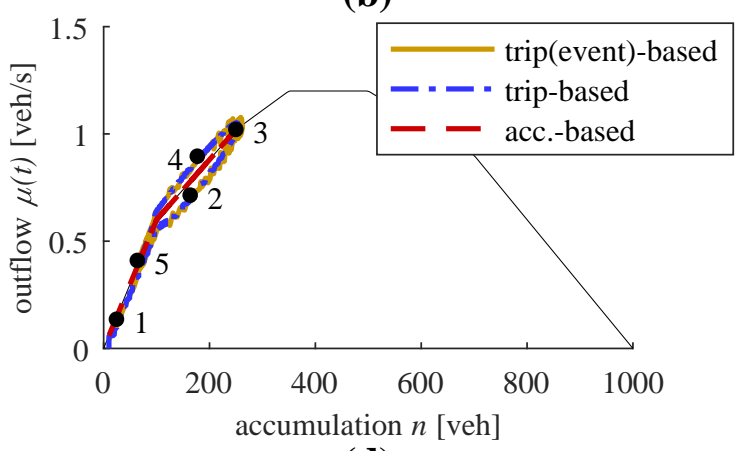

(d)

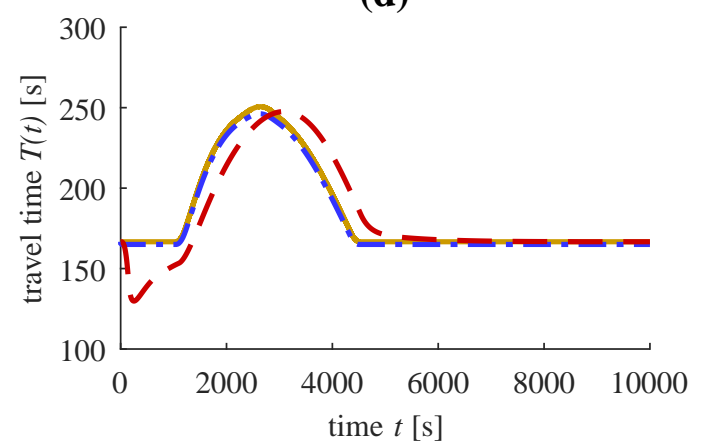

(f)

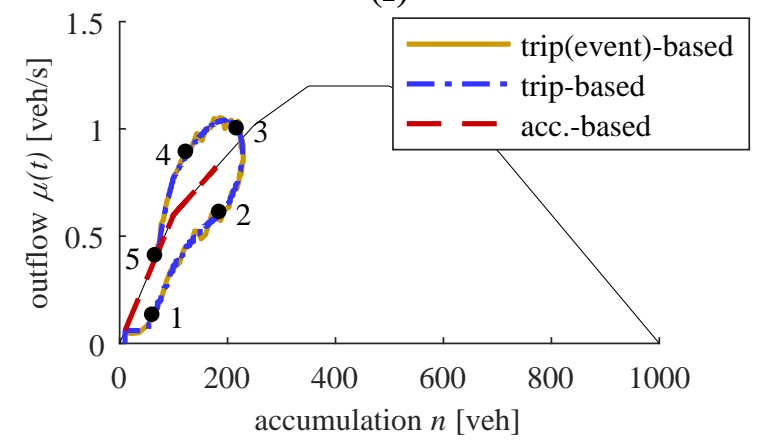

(h)

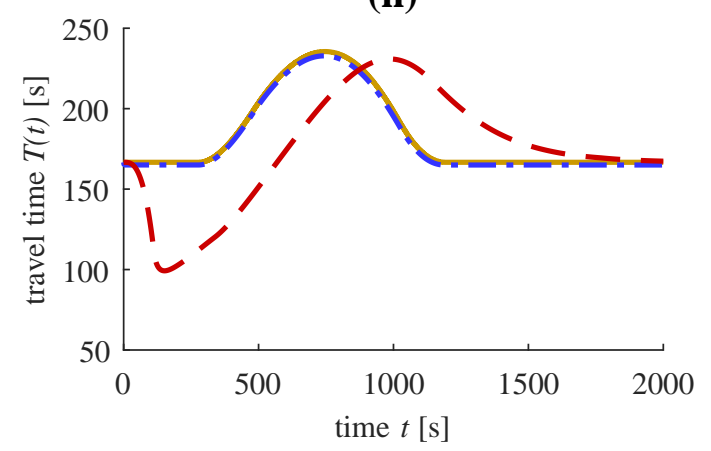

Figure 9: Comparison between models on two simulations. Simulation 1 with a slow-varying demand over 10,000 s: (a) peak demand profile, (b) outflow vs accumulation at each time, (c) evolution of accumulation and (d) evolution of travel time. Simulation 2 with a fast-varying demand over $2000 \mathrm{~s}$ : (e) peak demand profile, (f) outflow vs accumulation at each time, (g) evolution of accumulation and (h) evolution of travel time. 


\subsection{Temporary supply restriction at exits}

This case study aims to analyze the effect of a temporary supply restriction at the reservoir exit. This supply restriction is followed by a demand decrease to retrieve an equilibrium in free-flow conditions. Two scenarios described in figures 10(a) and (e) are compared. The first one concerns a short supply reduction, and we see that the accumulationbased and the trip-based models are almost equivalent in these conditions, see figures 10(a)-(d). The second one is about a longer supply reduction, and results show very different conclusions depending on the hypotheses made for the accumulation-based model in congestion.

In figures 10(f)-(h), the green curves ("model 1") correspond to our basic assumption of an entrance function $I(n)$ equal to the outflow-MFD in congestion, which is consistent with a CTM-based approach for inflow management. In this case the accumulation-based model reaches an equilibrium point on the congested part of the outflow-MFD and remains here even when the boundary conditions become more favorable after $5000 \mathrm{~s}$. With this approach the system is very sensitive to a supply reduction: if it is short enough the reservoir will return to a free-flow state after the demand has decreased like in figures 10(a)-(d), but if it is longer than a critical value, i.e. the time required to reach the congested part of the outflow-MFD, then the system state is permanently degraded. This situation may be even worse if no inflow limitation $I(n)$ is taken into account. The results in that case are represented by the red curves ("model 2"), after hitting the congested branch the accumulation-based model immediately converges to global gridlock. The last option corresponds to the yellow curves ("model 3"), it assumes an outflow-MFD which is not restricted in congestion, i.e. $O(n)$ is constant equal to the outflow capacity for high values of accumulation. This hypothesis allows a quasi-perfect matching between the accumulation-based and the trip-based models. In fact, the way we implemented congestion management in the trip-based approach in using Newell's method makes our reservoir behaving like a freeway in congestion. Then it is no surprise that its equivalent for the accumulation-based model is a CTM-like modeling approach. 
(a)

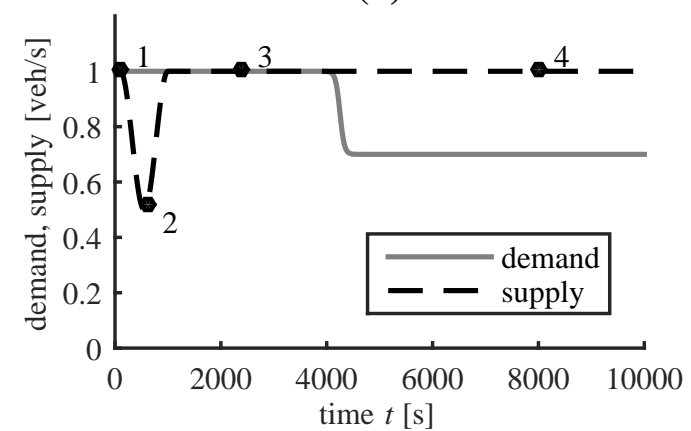

(c)

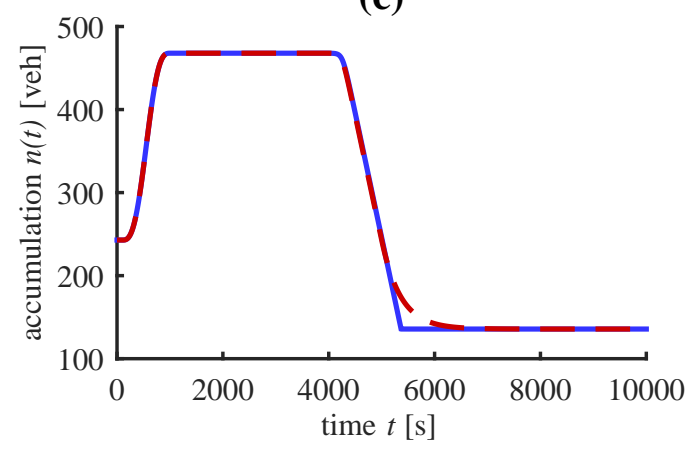

(e)

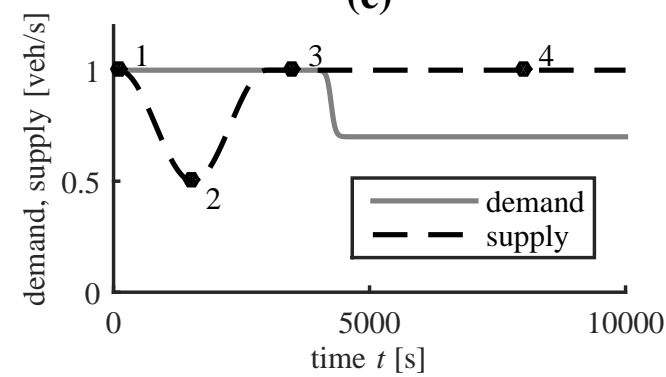

(g)

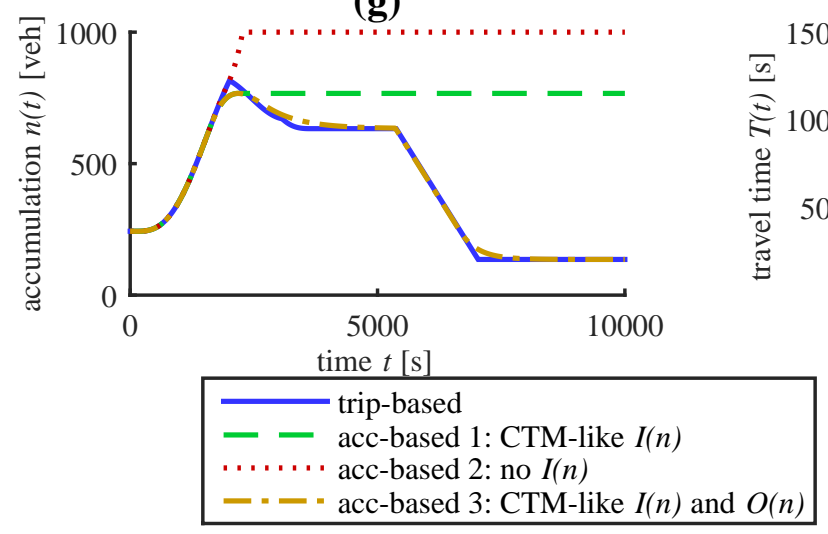

(b) $=\begin{gathered}\text { trip-based } \\ \text { acc.-based }\end{gathered}$

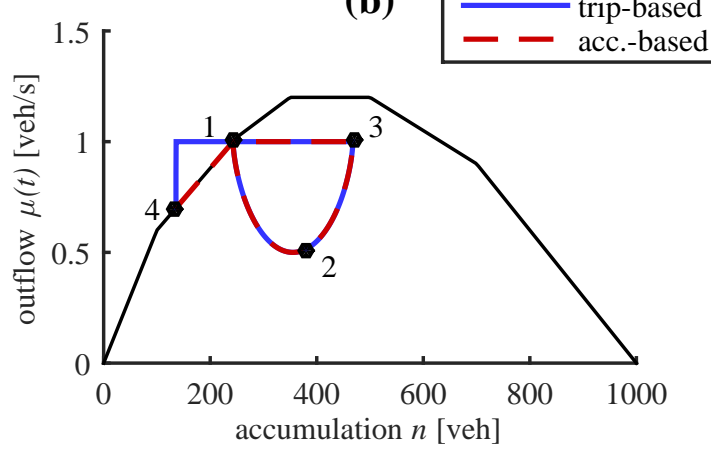

(d)

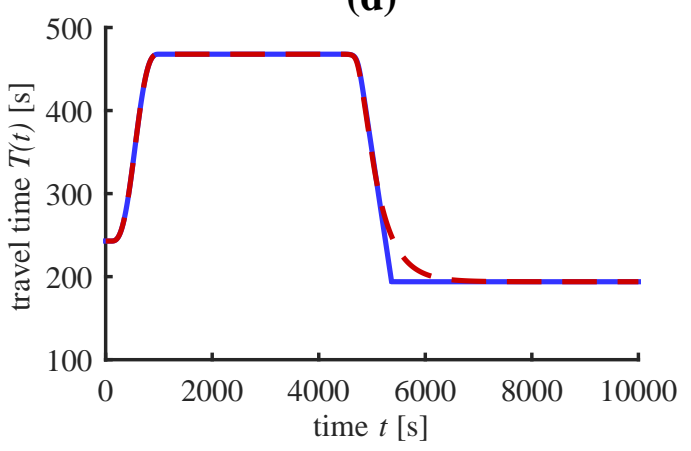

(f)

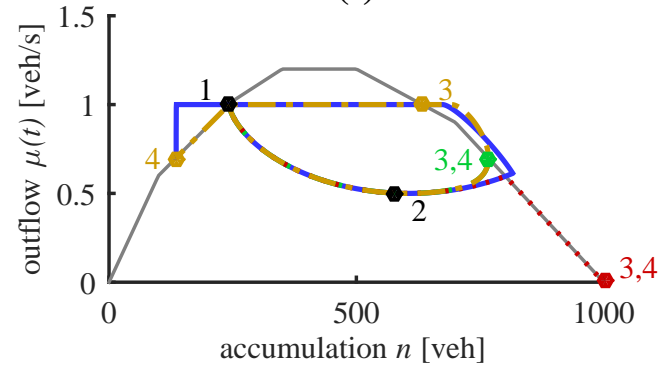

(h)

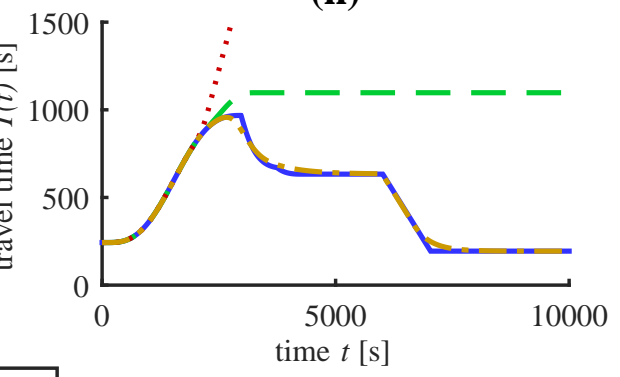

Figure 10: Comparison between models on two simulations. Simulation 1 with supply reduction over $1000 \mathrm{~s}$ : (a) demand and supply profiles, (b) outflow vs accumulation at each time, (c) evolution of accumulation and (d) evolution of travel time. Simulation 2 with supply reduction over $3000 \mathrm{~s}$, three cases for the accumulation-based model: 1, CTM-like $I(n)$, 2, no $I(n)$ and 3, CTM-like $I(n)$ and $O(n)$. (e) Demand and supply profiles, (f) outflow vs accumulation at each time, (g) evolution of accumulation and (h) evolution of travel time. 


\subsection{Case of different trip lengths}

This last case study is taking advantage of the event-based resolution method for the trip-based model to account for different groups of travelers having their own trip length. This is compared with an approach proposed by Geroliminis (2015) to include different trip length classes in the accumulation-based framework. This author considers $N$ classes of travelers, each class having a current accumulation $n_{i}(t)$ and a trip length $L_{i}, 1 \leq i \leq N$. The total accumulation is $n=\sum_{i=1}^{N} n_{i}$. A distinction is made for the outflow of each class, which means the system dynamics is described by the following equations in free-flow conditions:

$$
\forall i \in\{1, \ldots, N\}, \quad \frac{d n_{i}}{d t}=\lambda_{i}(t)-O_{i}\left(n_{i}(t)\right)
$$

where $\lambda_{i}(t)$ and $O_{i}\left(n_{i}(t)\right)$ are respectively the demand and the outflow for class $i$. We suppose the sum of all demands is below the global outflow capacity and no supply at exit is limiting the sum of all outflows. Otherwise a merge function has to be employed to manage inflows and outflows.

To calculate the average trip length $L$, note that it satisfies $L=n V(n) / O(n)$ and that $O(n)=\sum_{i=1}^{N} O_{i}\left(n_{i}\right)$, where each outflow function $O_{i}\left(n_{i}\right)$ is determined by applying Little's formula (Little, 1961): $O_{i}\left(n_{i}\right)=n_{i} V(n) / L_{i}$. Combining these equations, gives:

$$
L(t)=\frac{n(t)}{\sum_{i=1}^{N} \frac{n_{i}(t)}{L_{i}}}
$$

which is time-dependent since $n_{i}=n_{i}(t)$. An alternative definition can be obtained by noting that $\sum_{i=1}^{N} O_{i} L_{i}=n V(n)$ and therefore:

$$
L(t)=\frac{\sum_{i=1}^{N} O_{i}\left(n_{i}(t)\right) L_{i}}{O(n(t))}
$$

which means that the average trip length is also the arithmetic mean of all $L_{i}$ weighted by their corresponding outflow $O_{i}$.

Figures 11(a)-(d) present a situation with two classes: $L_{1}=1000 \mathrm{~m}$ and $L_{2}=2000 \mathrm{~m}$ with the same initial demand. A peak for the first class occurs at $t=500 \mathrm{~s}$. For the trip-based model the evolution of the average trip length is calculated by successive means of individual trip lengths over a given time period. It is the discretized version of $L(t)$, seen as the mean of all $L_{i}$ weighted by outflows. We expect a temporary drop in the evolution of $L(t)$, due to the demand peak for the smallest trip distance $L_{1}$. This should result in a higher outflow for this class, and thus a greater impact in the mean calculation for $L(t)$ compared to those traveling a longer distance $L_{2}$. On the whole the two modeling approaches reproduce this effect quite well, although the accumulation-based model emphasizes it more than the trip-based one, see figure 11(b). As regards the accumulation and travel time evolution, there is a clear overestimation of the demand peak for the accumulation-based model.

This study has been compared to a demand peak case with a unique trip length presented in figures 11(e)-(h). The demand profile $\lambda(t)$ is exactly the global demand of the previous case: $\lambda(t)=\lambda_{1}(t)+\lambda_{2}(t)$. Two situations are examined, one considers a constant trip length $L_{0}=1500 \mathrm{~m}$ equal to the mean of $L_{1}$ and $L_{2}$ in steady state, whereas the other concentrates on a time-varying trip length $L(t)$ which corresponds to the instantaneous mean of $L_{1}$ and $L_{2}$ at each time, i.e. $L(t)=\left(\lambda_{1}(t) L_{1}+\lambda_{2}(t) L_{2}\right) / \lambda(t)$.

We observe in figure 11(f) that $L(t)$ reproduces almost the same evolution as the previous one in figure 11(b). However, the behavior of accumulation and travel time is completely different in this case. As seen in figures $11(\mathrm{~g})$ and $(\mathrm{h})$, using either a constant or dynamic trip length $L(t)$ leads to an underestimation of the demand peak for the accumulation-based model as it was already noticed in section 4.1. However, if we assume that the most accurate modeling for this two-class problem is the trip-based approach with $L_{1}$ and $L_{2}$, then the accumulation-based model paradoxically gives better results in accumulation with $L_{0}$ or $L(t)$ than with $L_{1}$ and $L_{2}$. In this case, we have to compare the evolution of total $n(t)$ in figure $11(\mathrm{c})$ with $n(t)$ in figure $11(\mathrm{~g})$. We observe that the overestimation of the accumulation peak given by the accumulation-based model is lower in figure $11(\mathrm{~g})$ than in figure 11(c). 


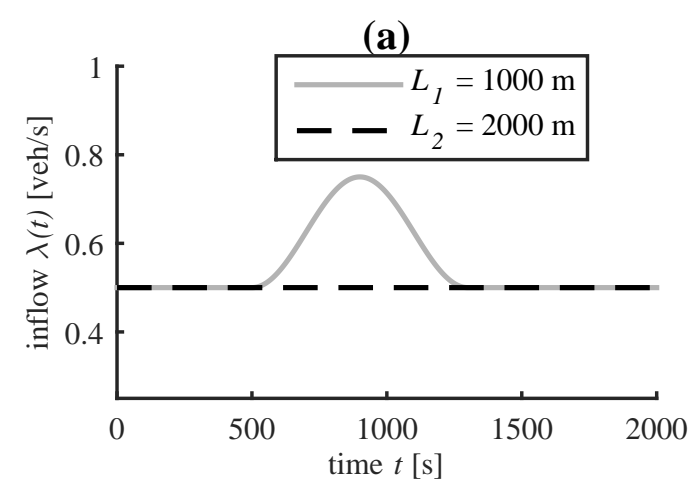

(c)

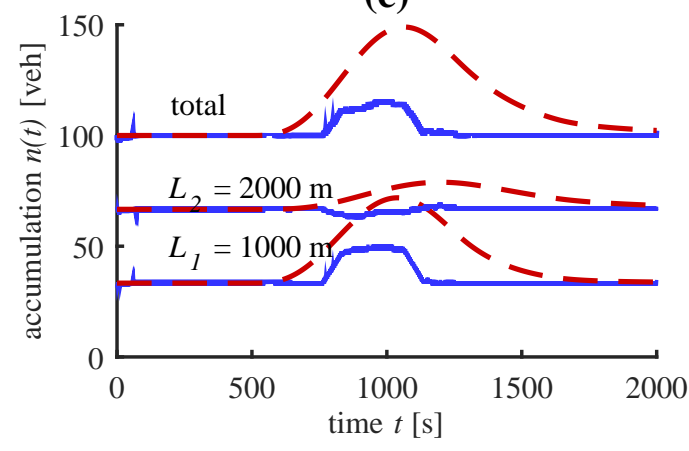

(e)

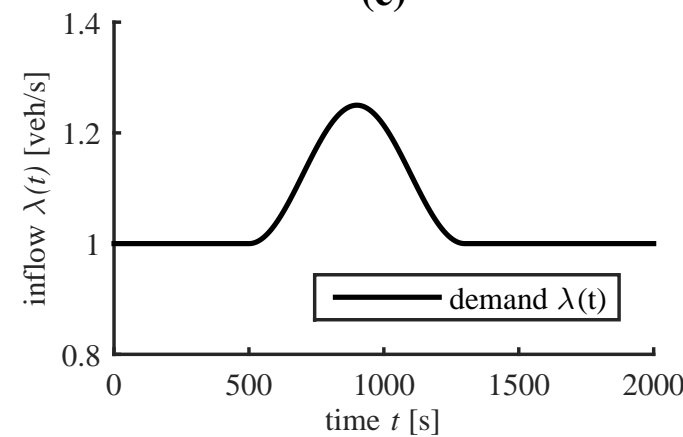

(g)

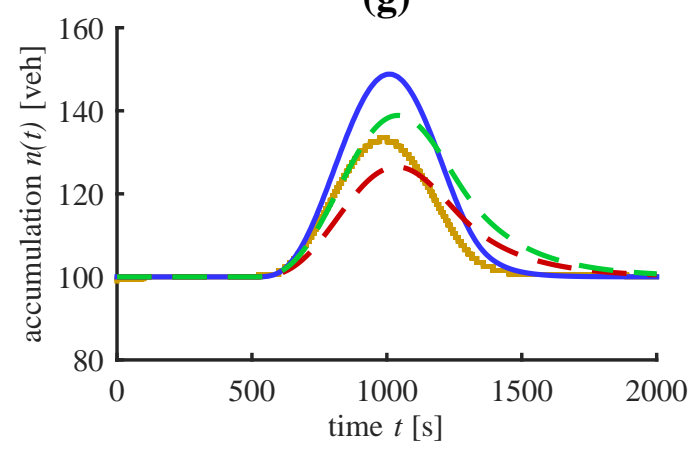

(b)

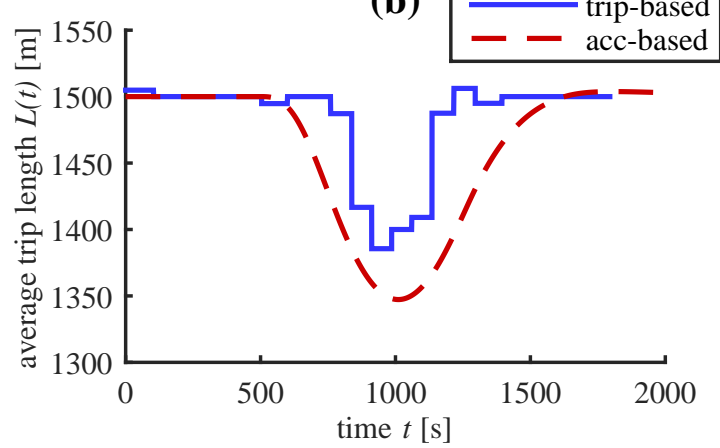

(d)

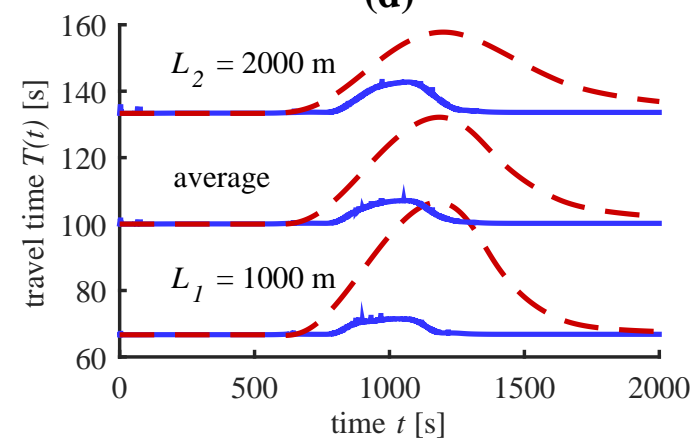

(f)

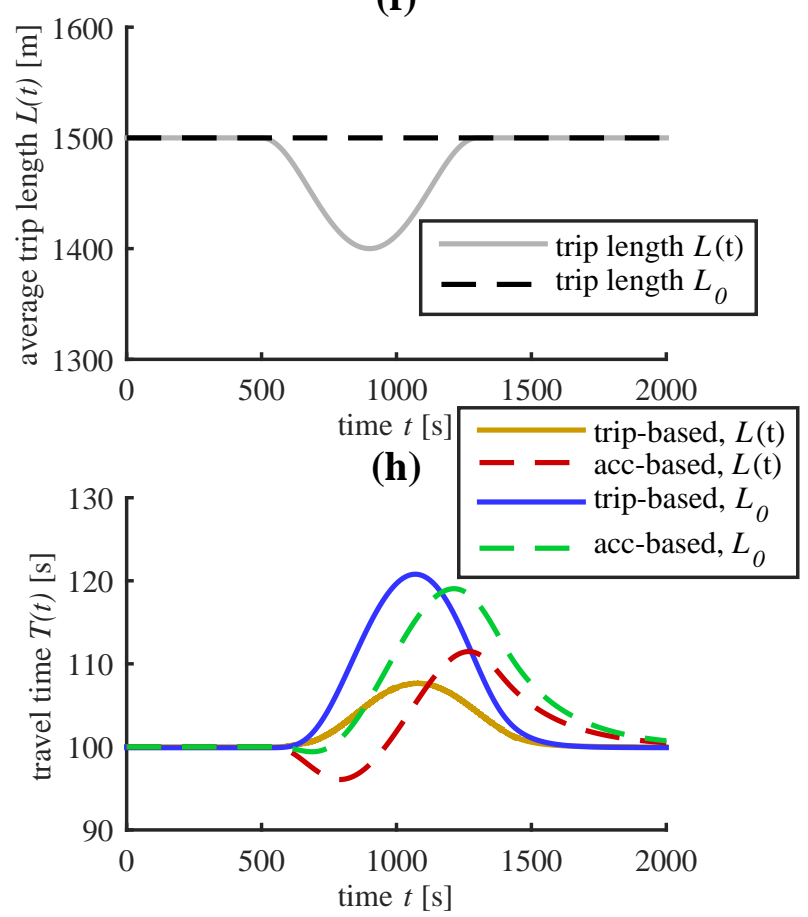

Figure 11: Comparison between models on two simulations. Simulation 1 with traveler trip lengths separated by class: (a) demand profile for each class, (b) evolution of the average trip length, (c) evolution of accumulation and (d) travel time for each class. Simulation 2 with a unique trip length varying or not through time: (e) demand profile, (f) evolution of the trip length, (g) evolution of accumulation and (h) travel time. 


\section{Discussion and conclusion}

In this paper we proposed different resolution schemes for the accumulation-based and the trip-based models. For the first one, exact solutions have been established with piecewise linear functions thanks to the semi-analytical method, although this process may be limited when changes in demand are numerous. The full numerical scheme proved to have acceptable numeric errors as well, and is more flexible than the semi-analytical method. Its basic formulation has also been extended to solve the approach of Geroliminis (2015) integrating multiple trip lengths. As for the trip-based framework, in comparison with Arnott (2013); Fosgerau (2015); Daganzo and Lehe (2015); Lamotte and Geroliminis (2016) we developed a numerical resolution method that can approximate the model solution in an efficient way, and can offer some theoretical support to our second resolution scheme. While having some similarities with the methods presented by the previous authors, the latter is event-based like in Lamotte and Geroliminis (2016), and does not introduce any numerical bias by the choice of a time step. With encouraging applications and results, it proved to be a very simple and convenient way to account for varying trip lengths among travelers, and thus may be considered as a promising tool for further applications like multi-reservoir modeling, studies including on-street parking search, or integration with route choice problems.

Apart from these consideration about resolution methods, this paper made a large comparison between the tripbased model and the accumulation-based one, which is still widely used for the development of more complex applications like multi-reservoir simulation and dynamic control. Our literature review allows to draw a synthesis about the hypothesis history of both approaches. Their differences are then clearly stated: unlike the trip-based model the accumulation-based one (i) mostly ignores traveled distances, (ii) has no memory of the past of the system, and (iii) considers that accumulation remains constant when a user travels in the reservoir. These assumptions are responsible for the incoherence in the model results during transient phases: drop in travel time when the demand increases and slow response of the system in fast-varying conditions are notably observed. In steady state however, the two models give the same results. Regarding the travel time drop phenomenon, we have then proposed a validity domain of the accumulation-based model with respect to the demand variation rate. We have also provided error estimation depending on the size of the demand variations. It should be noted that such errors correspond to simulation where the demand is determined from the beginning. Control applications for which the accumulation-based model is widely used introduce a feedback on this demand with respect to the reservoir state. Such feedback loop may certainly help to correct the mistakes of the accumulation-based model and then reduce the inaccuracies. On the other hand, multireservoir simulation grounded on the accumulation-based framework may be more obviously affected by the artifacts we highlighted.

\section{Acknowledgements}

This project has received funding from the European Research Council (ERC) under the European Union's Horizon 2020 research and innovation programme (grant agreement No 646592 - MAGnUM project).

\section{Appendix A. Trip-based model resolution at higher orders}

We use here the notations described in section 3.1 and on figure 5. As the vehicle $N$ and the vehicle $N+\delta N$ have the same trip length $L$, the definition in equation 8 allows us to write:

$$
\int_{t_{N}^{\text {in }}}^{t_{N}^{\text {out }}} V(n(t)) d t=L=\int_{t_{N+\delta N}^{\text {in }}}^{t_{N+\delta N}^{\text {out }}} V(n(t)) d t
$$

Splitting the integral limits on the left side of this equation in $\left[t_{N}^{\mathrm{in}}, t_{N+\delta N}^{\mathrm{in}}\right]=\left[t_{0}, t_{0}+\delta N / \lambda\left(t_{0}\right)\right]$ and $\left[t_{N+\delta N}^{\mathrm{in}}, t_{N}^{\text {out }}\right]$, and these on the right side in $\left[t_{N+\delta N}^{\text {in }}, t_{N}^{\text {out }}\right]$ and $\left[t_{N}^{\text {out }}, t_{N+\delta N}^{\text {out }}\right]=\left[t_{1}, t_{1}+\delta t\right]$ simplifies the equation to:

$$
\int_{t_{0}}^{t_{0}+\delta N / \lambda\left(t_{0}\right)} V(n(t)) d t=\int_{t_{1}}^{t_{1}+\delta t} V(n(t)) d t
$$

where $V(n)$ is given by the branch $P_{i}$ of the production-MFD, $V(n)=P_{i}(n) / n=w_{i}\left(1-\eta_{i} / n\right)$ on the interval $\left[t_{0}, t_{0}+\right.$ $\left.\delta N / \lambda\left(t_{0}\right)\right]$; and by the branch $P_{j}, V(n)=P_{j}(n) / n=w_{j}\left(1-\eta_{j} / n\right)$ on the interval $\left[t_{1}, t_{1}+\delta t\right]$. In order to solve 
equation A.2 in $\delta t$, we choose to approximate $V(n(t))$ by its Taylor polynomial at a given order. Then the resolution of the integrals should give polynomial expressions in $\delta t$. Let us start with order 2, if $\delta N$ is small enough we have for $t \in\left[t_{0}, t_{0}+\delta N / \lambda\left(t_{0}\right)\right]$ the following approximation:

$$
V(n(t)) \approx V\left(n_{0}\right)+\frac{\partial V}{\partial n}\left(n_{0}\right) \cdot\left(n(t)-n_{0}\right)+\frac{\partial^{2} V}{\partial n^{2}}\left(n_{0}\right) \cdot \frac{\left(n(t)-n_{0}\right)^{2}}{2}
$$

where $n_{0}=n\left(t_{0}\right)$. For $\delta t$ small enough, which should be the case if $\delta N$ is small, a similar approximation of $V(n(t))$ can be written for $t \in\left[t_{1}, t_{1}+\delta t\right]$ in replacing $t_{0}$ by $t_{1}$ and $n_{0}$ by $n_{1}=n\left(t_{1}\right)$. The differences in accumulation $\left(n(t)-n_{0}\right)$ and $\left(n(t)-n_{1}\right)$ can be expressed in assuming a linear variation of accumulation on each interval. With the simplified notations $\lambda_{0}=\lambda\left(t_{0}\right), \mu_{0}=\mu\left(t_{0}\right)$ and $\lambda_{1}=\lambda\left(t_{1}\right)$ we can write:

$$
n(t)= \begin{cases}n_{0}+\left(\lambda_{0}-\mu_{0}\right)\left(t-t_{0}\right) & \text { for } t \in\left[t_{0}, t_{0}+\delta N / \lambda_{0}\right] \\ n_{1}+\left(\lambda_{1}-\frac{\delta N}{\delta t}\right)\left(t-t_{1}\right) & \text { for } t \in\left[t_{1}, t_{1}+\delta t\right]\end{cases}
$$

which gives the two approximations of $V(n(t))$ on the two intervals:

$$
V(n(t))= \begin{cases}V_{0}+\frac{w_{i}-V_{0}}{n_{0}}\left(\lambda_{0}-\mu_{0}\right)\left(t-t_{0}\right)-\frac{w_{i}-V_{0}}{n_{0}^{2}}\left(\lambda_{0}-\mu_{0}\right)^{2}\left(t-t_{0}\right)^{2} & t \in\left[t_{0}, t_{0}+\delta N / \lambda_{0}\right] \\ V_{1}+\frac{w_{j}-V_{1}}{n_{1}}\left(\lambda_{1}-\frac{\delta N}{\delta t}\right)\left(t-t_{1}\right)-\frac{w_{j}-V_{1}}{n_{1}^{2}}\left(\lambda_{1}-\frac{\delta N}{\delta t}\right)^{2}\left(t-t_{1}\right)^{2} & t \in\left[t_{1}, t_{1}+\delta t\right]\end{cases}
$$

where $V_{0}=V\left(n_{0}\right)$ and $V_{1}=V\left(n_{1}\right)$. At order 0 for $V(n(t))$ the latter expressions simplify to the constant terms, and in this case the solution in $\delta t$ after integrating on both intervals is straightforward (order 1 for $\delta t$ ):

$$
\delta t=\frac{V_{0}}{V_{1}} \cdot \frac{\delta N}{\lambda_{0}}
$$

At order 1 for $V(n(t))$ the approximations of $V(n(t))$ are reduced to the constant terms and the terms in $t$, which gives after integration a second-order polynomial in $\delta t$ to solve (order 2 for $\delta t$ ):

$$
\lambda_{1}\left(V_{1}-w_{j}\right) \cdot \delta t^{2}-\left(2 n_{1} V_{1}+\left(V_{1}-w_{j}\right) \delta N\right) \cdot \delta t+2 n_{1}\left(V_{0} \frac{\delta N}{\lambda_{0}}-\frac{V_{0}-w_{i}}{2 n_{0}}\left(1-\frac{\mu_{0}}{\lambda_{0}}\right) \frac{\delta N^{2}}{\lambda_{0}}\right)=0
$$

Note that when $V_{0}=w_{i}$ and $V_{1}=w_{j}$, which is possible only if $w_{i}=w_{j}$ is the free-flow speed (i.e. $i=j=1$, we are on the first branch of the MFD), equation A.7 reduces to equation A.6. In a similar way, order 2 for $V(n(t))$ will provide a third-order polynomial in $\delta t$ which is not mentioned here.

Comparisons between the calculations of $\delta t$ at different orders are presented in figures A.12(a)-(c). These calculations have been carried on with different values for the accumulation $n_{1}$ at time $t_{1}$ (from 30 to 800 veh) and for the demand ratio $\lambda_{1} / \lambda_{0}$ (from 0.5 to 2 ). In each case we have chosen $n_{1}-n_{0}=20$ veh and $\lambda_{1}=0.5 \mathrm{veh} / \mathrm{s}$. Slight differences between the choices of order are only noticed for a high value of $\delta N(10 \mathrm{veh})$. This means for a reasonable value of $\delta N$ (e.g. $0.1 \mathrm{veh}$ ), the order 0 (i.e. the order 1 for $\delta t$ ) is largely sufficient for our resolution scheme.

\section{Appendix B. Numerical investigations for a parabolic MFD with dimensionless parameters}

We provide here some insights about a possible generalization of some of our conclusions about the accumulationbased model. For this purpose, we use a parabolic (Greenshield) MFD and the following dimensionless variables:

- $n^{*}=n / n_{j}$ : the accumulation $n$ to the maximum jam accumulation $n_{j}$ ratio

- $t^{*}=t / t_{\text {free }}$ : the time to the reservoir free-flow travel time $t_{\text {free }}=L / u$ ratio, where $u$ is the free-flow speed.

- $\lambda^{*}=\lambda / \mu_{c}$ : the demand to the outflow-MFD capacity $\mu_{c}=P_{c} / L$ (maximum outflow) ratio, where $P_{c}$ is the production-MFD capacity

We use also two dimensionless parameters:

- $\rho=\lambda_{0} / \mu_{c}$ : the initial demand to the outflow-MFD capacity $\mu_{c}$ ratio 
(a)

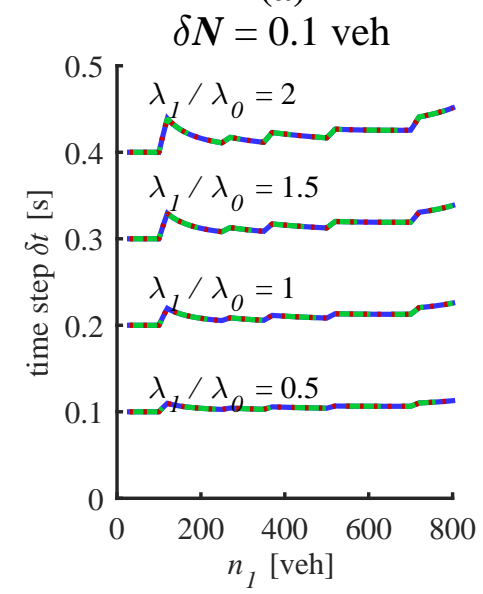

(b)

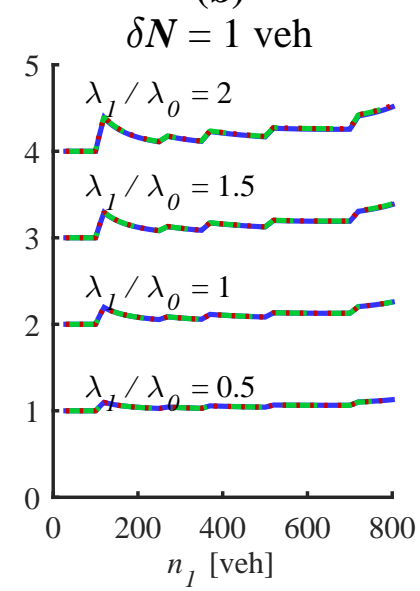

(c)

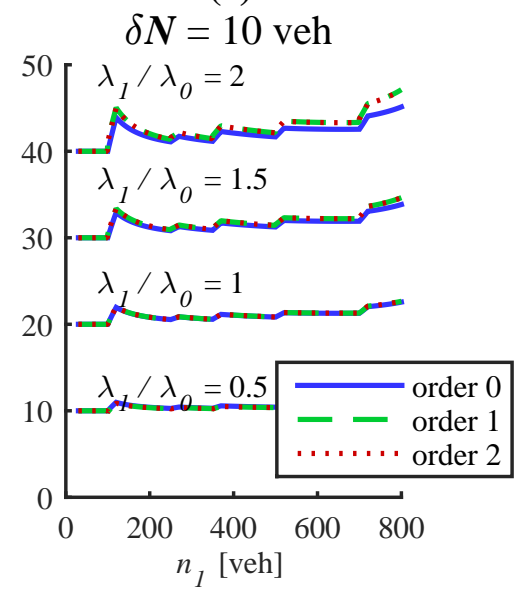

Figure A.12: Values of the time step $\delta t$ calculated at different orders for $V(n(t))$ and for different accumulation $n_{1}$ and ratio of demand level $\lambda_{1} / \lambda_{0}$. The difference $n_{1}-n_{0}$ is equal to $20 \mathrm{veh}$ in each case and $\lambda_{1}=0.5 \mathrm{veh} / \mathrm{s}$. (a) Case of a vehicle increment of $\delta N=0.1 \mathrm{veh},(\mathrm{b}) \delta N=1 \mathrm{veh}$, and (c) $\delta N=10$ veh

- $\beta=t_{\mathrm{dem}} / t_{\text {free }}$ : the demand relaxation time $t_{\mathrm{dem}}$ to the MFD free-flow travel time $t_{\text {free }}$ ratio

Laval and Castrillon (2015); Laval and Chilukuri (2016) showed that under linear transformation of flow, accumulation, space and time, delays and other performance indicators are invariant. This allows the simplification of any problem to a symmetric MFD shape case. That is why we choose here a simple parabolic MFD, which characteristic parameters are normalized by the transformations mentioned above. Hence the results presented in this appendix are intending to be universally applicable.

Figure B.13 illustrates the influence of the number of steps used to approximate a continuous demand profile. We choose here an exponential function:

$$
\lambda(t)=\lambda_{0}+\left(\mu_{c}-\lambda_{0}\right) \cdot\left(1-\mathrm{e}^{-t / t_{\mathrm{dem}}}\right)
$$

which in dimensionless form reads:

$$
\lambda^{*}\left(t^{*}\right)=\rho+(1-\rho) \cdot\left(1-\mathrm{e}^{-t^{*} / \beta}\right)
$$

Here the parameter $\rho$ is chosen equal to 0.1 and $\beta$ equal to 1.5 . The solutions provided by our semi-analytical process are compared with a full analytical solution obtained with Mathematica ${ }^{\circledR}$ for this kind of demand. The detailed analytical expression can be found in Laval et al. (2017). Results show that if the number of demand steps is high enough for our semi-analytical resolution (e.g. more than 40), the solution we get is very close to the exact solution. On the other hand a few branches (around 8) are sufficient to get good approximation of the MFD shape.

Figure B.14 also illustrates the influence of the time step $\delta t$ choice for the full numerical resolution, in comparison with the full analytical one from Mathematica ${ }^{\circledR}$ for the same demand case. It is observed that a timestep small enough leads to acceptable approximation of the exact solution too, namely $\delta t$ should be less than $10 \%$ of the free-flow travel time $t_{\text {free }}$.

Figure B.15 presents our analysis in section 2.6 about the definition of a validity domain for the accumulationbased model applied to this dimensionless study. The demand profile is a logistic "S-shaped" function that is also characterized by the parameters $\rho$ and $\beta$ in dimensionless form. The demand gap $\Delta \lambda$ is normalized by the initial demand $\lambda_{0}$, and the travel time drop height $h_{0}$ and duration $d_{0}$ are normalized by the free-flow travel time. Hence the dimensionless demand variation rate $Q_{\lambda}^{*}$ is obtained from the actual variation rate $Q_{\lambda}$ with: $Q_{\lambda}^{*}=Q_{\lambda} \cdot t_{\text {free }} / \lambda_{0}$. If we focus only on the drop height artifact, a demand variation rate $Q_{\lambda}^{*}<0.1$ should ensure a drop height effect below $10 \%$ of the free-flow travel time, according to figure B.15(c). In fact these charts cannot be used for any demand profile case, as these results are still dependent on $\rho$ (or $\lambda_{0}$ ). Here we choose $\rho=0.1$, but we have verified that the drop height $h_{0}$ seems to be independent of $\rho$. This work is currently under research. 
(a)

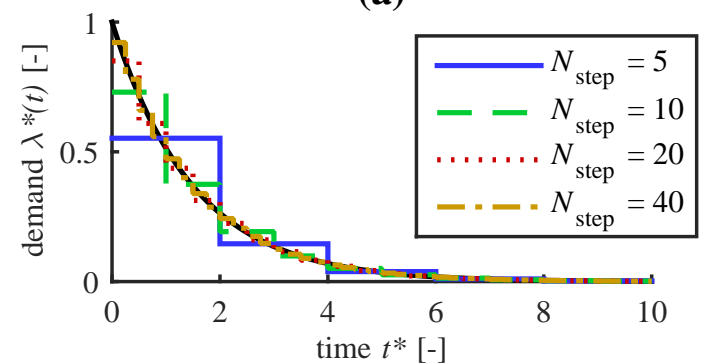

(c)

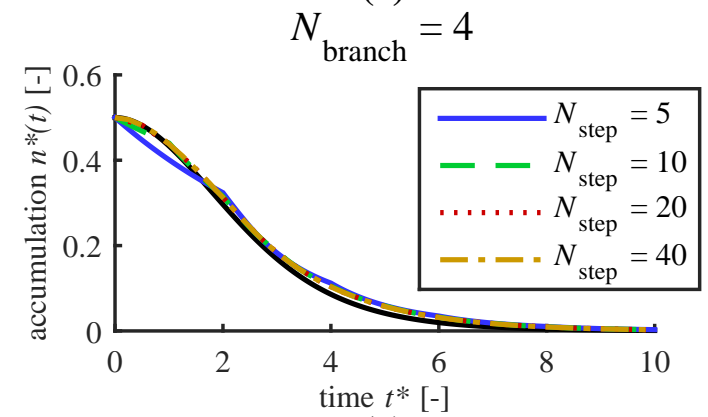

(e)

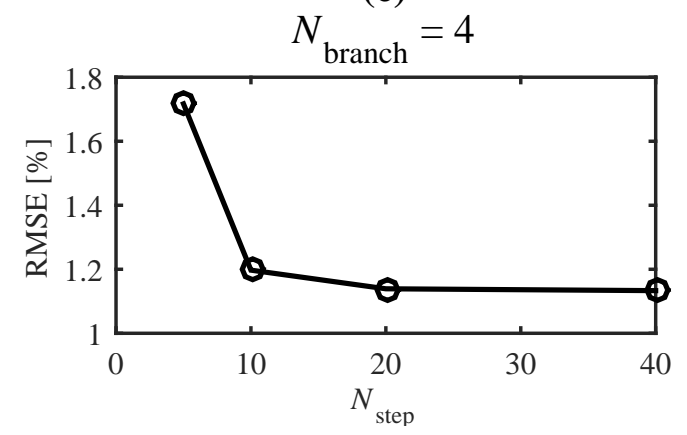

(b)

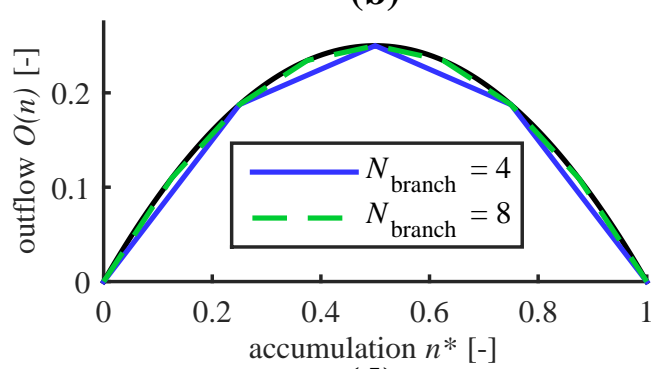

(d)

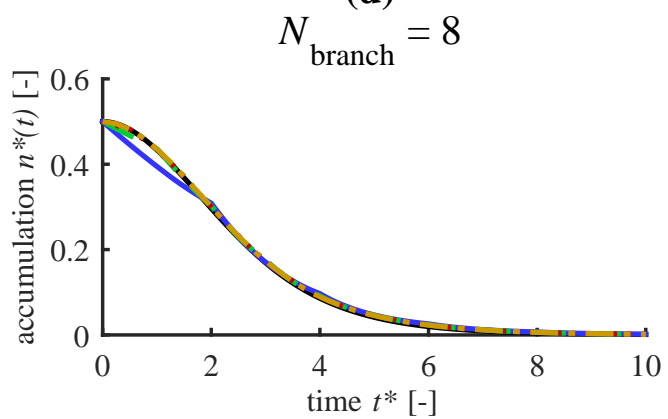

(f)

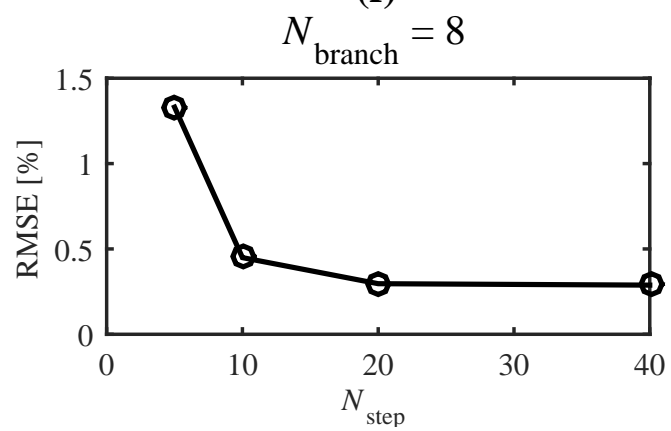

Figure B.13: Convergence test for the semi-analytical method. (a) Exponential demand profile discretization, (b) parabolic MFD discretization, (c) evolution of accumulation for a 4-branch and (d) a 8-branch MFD, (e) root mean square error vs number of demand steps for a 4-branch and (f) a 8-branch MFD. The parameter $\rho$ is chosen equal to 0.1 and $\beta$ equal to 1.5 .

(a)

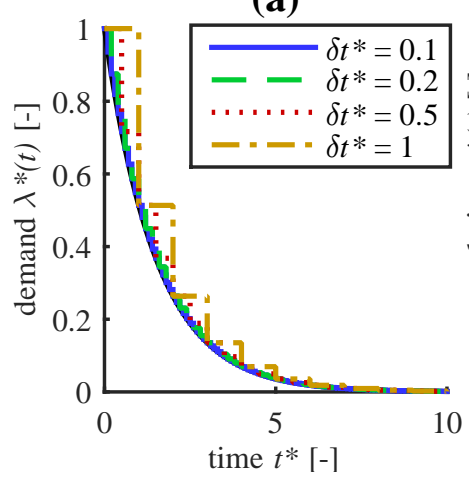

(b)

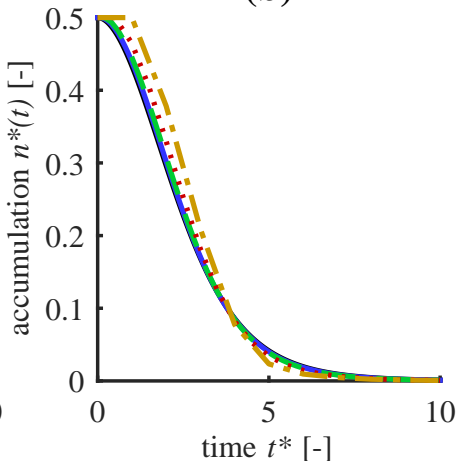

(c)

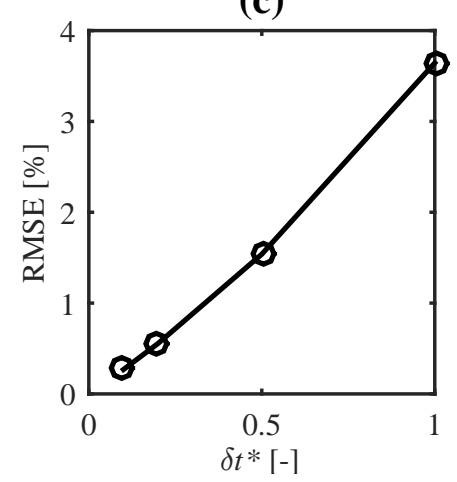

Figure B.14: Convergence test for the full numerical scheme. (a) Exponential demand profile discretization, (b) evolution of accumulation and (c) root mean square error vs timestep 
(a)
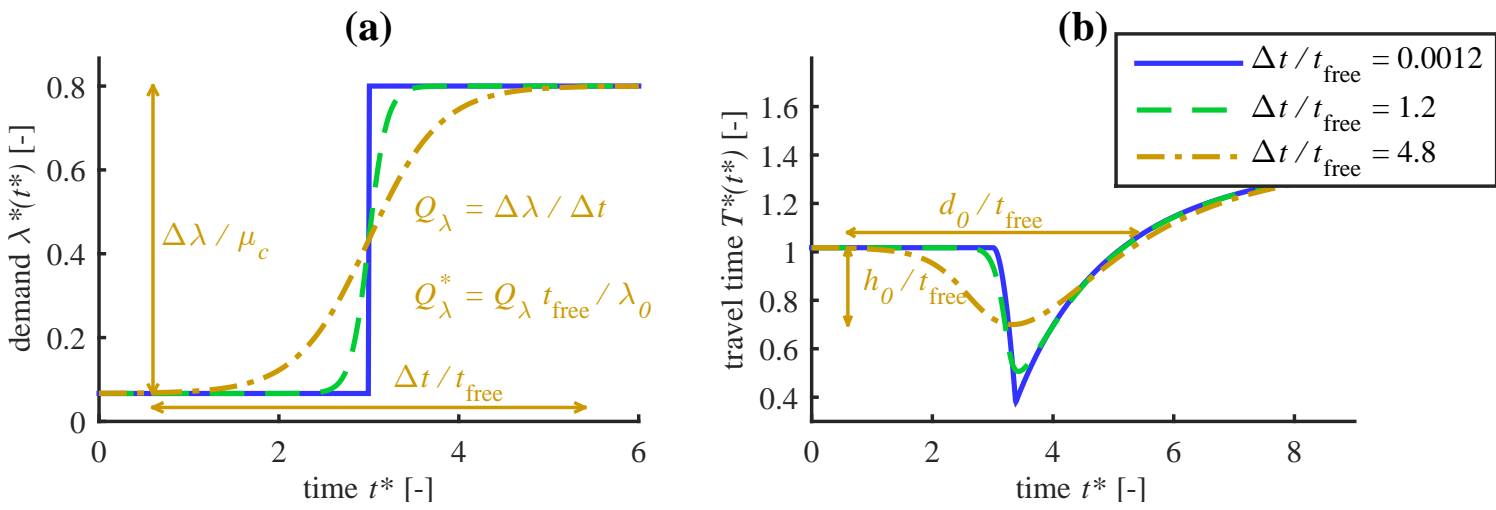

(c)

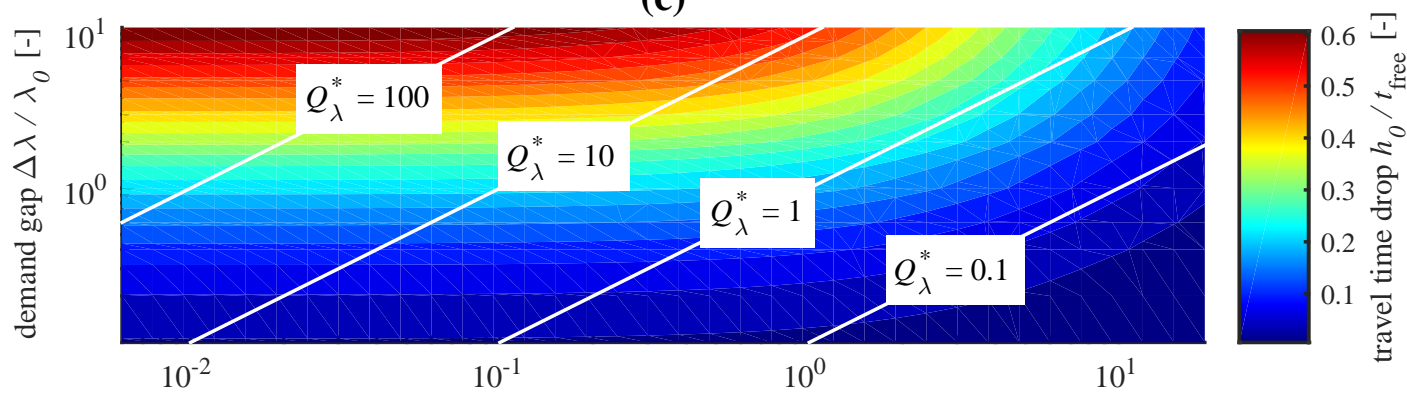

(d)

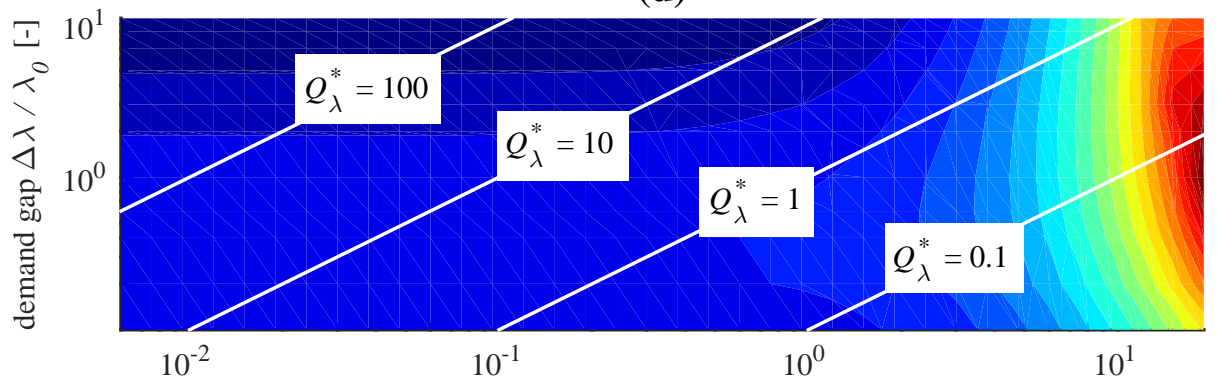

(e)

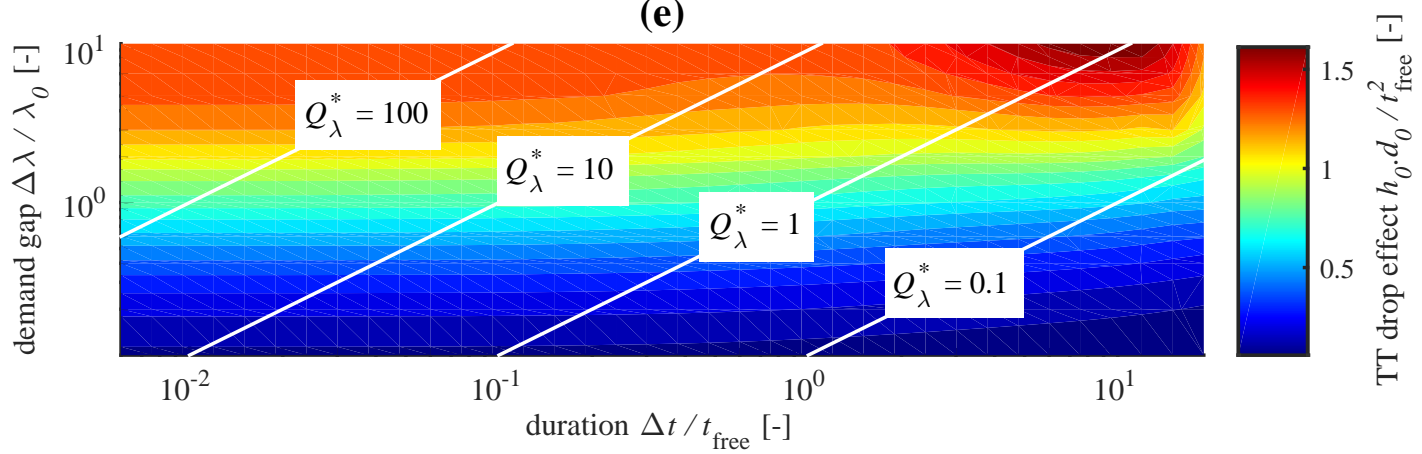

Figure B.15: Influence of the demand variation rate on the accumulation-based model accuracy for travel time. This accuracy is defined regarding the size of the travel time drop artifact. (a) Demand profiles with different variation rates, (b) resulting travel time profiles with the normalized drop height $h_{0} / t_{\text {free }}$ and drop duration $d_{0} / t_{\text {free }}$, (c) drop height value in the $\left(\Delta t^{*}, \Delta \lambda^{*}\right)$ space, (d) drop duration value, and (e) the product height-duration value in the same plane 


\section{Appendix C. Notation glossary}

Table C.1: General notations

\begin{tabular}{|c|c|}
\hline Notation & Definition [units] \\
\hline$t$ & Current time $[\mathrm{s}]$ \\
\hline$n(t), n$ & Accumulation $=$ number of vehicles circulating in the network at time $t$ [veh] \\
\hline$T(t)$ & Experienced travel time $=$ time spent in the reservoir by the vehicle exiting at time $t[\mathrm{~s}]$ \\
\hline$N_{\text {in }}(t)$ & Cumulative count curve for entering vehicles [veh] \\
\hline$N_{\text {out }}(t)$ & Cumulative count curve for exiting vehicles [veh] \\
\hline$P(n)$ & Production-MFD = travel production $[\mathrm{veh} . \mathrm{m} / \mathrm{s}]$ \\
\hline$V(n)$ & Speed-MFD $=$ vehicle mean speed in the reservoir $=P(n) / n[\mathrm{~m} / \mathrm{s}]$ \\
\hline$O(n)$ & Outflow-MFD $=$ outflow demand from the reservoir $=P(n) / L[\mathrm{veh} / \mathrm{s}]$ \\
\hline$I(n)$ & CTM-like entrance or supply function for inflow restriction at the reservoir entry [veh/s] \\
\hline$L$ & Reservoir average trip length (accumulation-based) or vehicle trip length (trip-based) [m] \\
\hline$u$ & Free-flow speed $[\mathrm{m} / \mathrm{s}]$ \\
\hline$t_{\text {free }}$ & Reservoir free-flow travel time $=L / u[\mathrm{~s}]$ \\
\hline$n_{j}$ & Reservoir maximum (jam) accumulation [veh] \\
\hline$P_{c}$ & Production-MFD capacity = maximum production $[\mathrm{veh} . \mathrm{m} / \mathrm{s}]$ \\
\hline$\mu_{c}$ & Outflow-MFD capacity $=$ maximum outflow $=P_{c} / L[\mathrm{veh} / \mathrm{s}]$ \\
\hline$\lambda(t)$ & Inflow into the reservoir $=$ inflow demand in free-flow $[\mathrm{veh} / \mathrm{s}]$ \\
\hline$\mu(t)$ & Outflow from the reservoir $[\mathrm{veh} / \mathrm{s}]$ \\
\hline$P_{i}(n)$ & $i$ th branch of the piecewise linear production-MFD (linear function) [veh.m/s] \\
\hline$w_{i}$ & Slope of the $i$ th branch $P_{i}(n)[\mathrm{m} / \mathrm{s}]$ \\
\hline$\eta_{i}$ & $n$-intercept of the $i$ th branch $P_{i}(n)[\mathrm{veh}]$ \\
\hline$n_{i}$ & Accumulation value at the intercept of the $i$ th and the $(i+1)$ th branches of $P(n)$ [veh] \\
\hline$t_{i}$ & Time at which there is a change in MFD branch during the evolution of $n(t)$ or $T(t)[\mathrm{s}]$ \\
\hline$t_{d}$ & Time at which there is a change in demand value $[\mathrm{s}]$ \\
\hline$n_{\text {ini }}$ & Initial accumulation at $t=0$ [veh] \\
\hline$T_{\text {ini }}$ & Initial travel time at $t=0[\mathrm{~s}]$ \\
\hline$\lambda_{\text {ini }}$ & Initial demand at $t=0[\mathrm{veh} / \mathrm{s}]$ \\
\hline$n_{0}$ & $n\left(t_{0}\right)=$ Accumulation at time $t_{0}[\mathrm{veh}]$ \\
\hline$T_{0}$ & $T\left(t_{0}\right)=$ Travel time at time $t_{0}[\mathrm{~s}]$ \\
\hline$\lambda_{0}$ & Constant demand value, or $\lambda\left(t_{0}\right)=$ inflow at time $t_{0}[\mathrm{veh} / \mathrm{s}]$ \\
\hline$\mu_{0}$ & Constant supply value, or $\mu\left(t_{0}\right)=$ outflow at time $t_{0}[\mathrm{veh} / \mathrm{s}]$ \\
\hline$\delta t$ & Time step for the Euler-type finite difference resolution method [s] \\
\hline$\Delta \lambda$ & Inflow gap in the evolution of the demand $\lambda(t)[\mathrm{veh} / \mathrm{s}]$ \\
\hline$\Delta t$ & Duration of the inflow gap in the evolution of the demand $\lambda(t)$ [s] \\
\hline$Q_{\lambda}$ & Demand variation rate $=$ ratio of $\Delta \lambda$ over $\Delta t\left[\mathrm{veh} / \mathrm{s}^{2}\right]$ \\
\hline$h_{0}$ & Height of the travel time drop in the evolution of $T(t)[\mathrm{s}]$ \\
\hline$d_{0}$ & Duration of the travel time drop in the evolution of $T(t)[\mathrm{s}]$ \\
\hline
\end{tabular}


Table C.2: Additional notations from section 3

\begin{aligned} & \hline \hline Notation Definition [units] \\ & \hline$N$ Vehicle index [veh] \\ &$\delta N$ Vehicle fraction or increment (may be below 1) = discretization step of the vehicle index [veh] \\ &$t_{0}=t_{N}^{\text {in }}$ Entering time of vehicle $N$ [s] \\ &$t_{1}=t_{N}^{\text {out }}$ Exiting time of vehicle $N[\mathrm{~s}] \\ & t_{N+\delta N}^{\text {in }}$ Entering time of vehicle $N+\delta N[\mathrm{~s}] \\ & t_{N+\delta N}^{\text {out }}$ Exiting time of vehicle $N+\delta N[\mathrm{~s}] \\ & \delta t$ Elapsed time between the exits of vehicles $N$ and $N+\delta N=t_{N+\delta N}^{\text {out }}-t_{N}^{\text {out }}[\mathrm{s}] \\ & n_{0}, T_{0}, \lambda_{0}, \mu_{0}, V_{0}$ Respectively accumulation [veh], travel time [s], inflow [veh/s], outflow [veh/s] \\ & and mean speed [m/s] at time $t_{0}$ [veh] \\ &$n_{1}, T_{1}, \lambda_{1}, \mu_{1}, V_{1}$ Respectively accumulation [veh], travel time [s], inflow [veh/s], outflow [veh/s] \\ & and mean speed [m/s] at time $t_{1}[\mathrm{veh}] \\ &$\hline\end{aligned}




\section{References}

Aboudolas, K., Geroliminis, N., 2013. Perimeter and boundary flow control in multi-reservoir heterogeneous networks. Transportation Research Part B: Methodological 55, 265-281.

URL http://www.sciencedirect.com/science/article/pii/S0191261513001185

Arnott, R., 2013. A bathtub model of downtown traffic congestion. Journal of Urban Economics 76, 110-121.

URL http://www.sciencedirect.com/science/article/pii/S0094119013000107

Buisson, C., Ladier, C., 2009. Exploring the impact of homogeneity of traffic measurements on the existence of macroscopic fundamental diagrams. Transportation Research Record: Journal of the Transportation Research Board 2124, 127-136.

Daganzo, C. F., Jan. 2007. Urban gridlock: Macroscopic modeling and mitigation approaches. Transportation Research Part B: Methodological $41(1), 49-62$.

URL http: //www.sciencedirect.com/science/article/B6V99-4JWFGNC-2/1/4659a928f cc742053a9587464572f409

Daganzo, C. F., 2011. On the macroscopic stability of freeway traffic. Transportation Research Part B: Methodological 45 (5), $782-788$. URL http://www.sciencedirect.com/science/article/pii/S0191261511000154

Daganzo, C. F., Lehe, L. J., 2015. Distance-dependent congestion pricing for downtown zones. Transportation Research Part B: Methodological 75, 89-99.

URL http: //www.sciencedirect.com/science/article/pii/S0191261515000387

Fosgerau, M., 2015. Congestion in the bathtub. Economics of Transportation 4 (4), 241-255. URL http: //www.sciencedirect.com/science/article/pii/S2212012215000398

Gayah, V. V., Daganzo, C. F., 2011. Clockwise hysteresis loops in the macroscopic fundamental diagram: An effect of network instability. Transportation Research Part B: Methodological 45 (4), 643-655. URL http://www.sciencedirect.com/science/article/pii/S0191261510001396

Geroliminis, N., 2015. Cruising-for-parking in congested cities with an \{MFD\} representation. Economics of Transportation 4 (3), 156-165. URL http://www.sciencedirect.com/science/article/pii/S2212012215000180

Geroliminis, N., Daganzo, C. F., 2007. Macroscopic modeling of traffic in cities. In: Transportation Research Board 86th Annual Meeting. 07-0413. Washington DC.

Geroliminis, N., Sun, J., 2011. Hysteresis phenomena of a macroscopic fundamental diagram in freeway networks. Transportation Research Part A: Policy and Practice 45 (9), 966-979, select Papers from the 19th International Symposium on Transportation and Traffic Theory (ISTTT). URL http://www.sciencedirect.com/science/article/pii/S0965856411000620

Haddad, J., 2017. Optimal perimeter control synthesis for two urban regions with aggregate boundary queue dynamics. Transportation Research Part B: Methodological 96, 1-25.

URL //www.sciencedirect.com/science/article/pii/S0191261516308025

Haddad, J., Geroliminis, N., 2012. On the stability of traffic perimeter control in two-region urban cities. Transportation Research Part B: Methodological 46 (9), 1159-1176.

URL http://www.sciencedirect.com/science/article/pii/S0191261512000641

Hajiahmadi, M., Knoop, V., De Schutter, B., Hellendoorn, H., Oct 2013. Optimal dynamic route guidance: A model predictive approach using the macroscopic fundamental diagram. In: Intelligent Transportation Systems - (ITSC), 2013 16th International IEEE Conference on. pp. 10221028 .

Knoop, V. L., Hoogendoorn, S. P., 2014. Network transmission model: a dynamic traffic model at network level. In: Transportation Research Board 93rd Annual Meeting. 14-1104. Washington DC.

Kouvelas, A., Saeedmanesh, M., Geroliminis, N., 2017. Enhancing model-based feedback perimeter control with data-driven online adaptive optimization. Transportation Research Part B: Methodological 96, 26-45.

URL http://www.sciencedirect.com/science/article/pii/S019126151630710X

Lamotte, R., Geroliminis, N., 2016. The morning commute in urban areas: Insights from theory and simulation. In: Transportation Research Board 95th Annual Meeting. 16-2003. Washington DC.

URL https: //trid.trb.org/view/1392730

Laval, J. A., Castrillon, F., 2015. Stochastic approximations for the macroscopic fundamental diagram of urban networks. Transportation Research Procedia 7, 615-630, 21st International Symposium on Transportation and Traffic Theory Kobe, Japan, 5-7 August, 2015. URL http://www.sciencedirect.com/science/article/pii/S2352146515001003

Laval, J. A., Chilukuri, B. R., 2016. Symmetries in the kinematic wave model and a parameter-free representation of traffic flow. Transportation Research Part B: Methodological 89, 168-177. URL http://www.sciencedirect.com/science/article/pii/S0191261515301016

Laval, J. A., Leclercq, L., Chiabaut, N., 2017. Dynamic traffic assignment using the macroscopic fundamental diagram: Freeway vs city streets user equilibrium revisited. In: Transportation Research Board 96th Annual Meeting. 17-04531. Washington DC.

Leclercq, L., Chiabaut, N., Trinquier, B., 2014. Macroscopic fundamental diagrams: A cross-comparison of estimation methods. Transportation Research Part B: Methodological 62, 1-12.

URL http://www.sciencedirect.com/science/article/pii/S0191261514000174

Leclercq, L., Parzani, C., Knoop, V. L., Amourette, J., Hoogendoorn, S. P., 2015. Macroscopic traffic dynamics with heterogeneous route patterns. Transportation Research Part C: Emerging Technologies 55, 292-307.

URL http: //www.sciencedirect.com/science/article/pii/S0968090X15001783

Little, J. D. C., May 1961. A proof for the queuing formula. Operations Research 9 (3), 383-387.

Mahmassani, H. S., Saberi, M., Zockaie, A., 2013. Urban network gridlock: Theory, characteristics, and dynamics. Transportation Research Part C: Emerging Technologies 36, 480-497.

URL http://www.sciencedirect.com/science/article/pii/S0968090X13001551 
Mariotte, G., Leclercq, L., 2016. Impact of the demand-supply distribution and the trip length choice on mfd-based traffic modeling. In: HEART 5th Annual Symposium. Delft.

Newell, G. F., 1993. A simplified theory of kinematic waves in highway traffic, part ii: Queueing at freeway bottlenecks. Transportation Research Part B: Methodological 27 (4), 289-303.

URL http://www.sciencedirect.com/science/article/pii/019126159390039D

Ramezani, M., Haddad, J., Geroliminis, N., 2015. Dynamics of heterogeneity in urban networks: aggregated traffic modeling and hierarchical control. Transportation Research Part B: Methodological 74, 1-19.

URL http://www.sciencedirect.com/science/article/pii/S0191261515000028

Xue, Z., Chiabaut, N., Leclercq, L., 2016. Evaluation of the effect of traffic modeling on the control of traffic networks. In: Transportation Research Board 95th Annual Meeting. 16-2205. Washington DC

URL https://trid.trb.org/view/1392820

Yildirimoglu, M., Geroliminis, N., 2014. Approximating dynamic equilibrium conditions with macroscopic fundamental diagrams. Transportation Research Part B: Methodological 70, 186-200.

URL http://www.sciencedirect.com/science/article/pii/S0191261514001568

Yildirimoglu, M., Ramezani, M., Geroliminis, N., 2015. Equilibrium analysis and route guidance in large-scale networks with \{MFD\} dynamics. Transportation Research Part C: Emerging Technologies 59, 404-420.

URL http://www.sciencedirect.com/science/article/pii/S0968090X15001813 